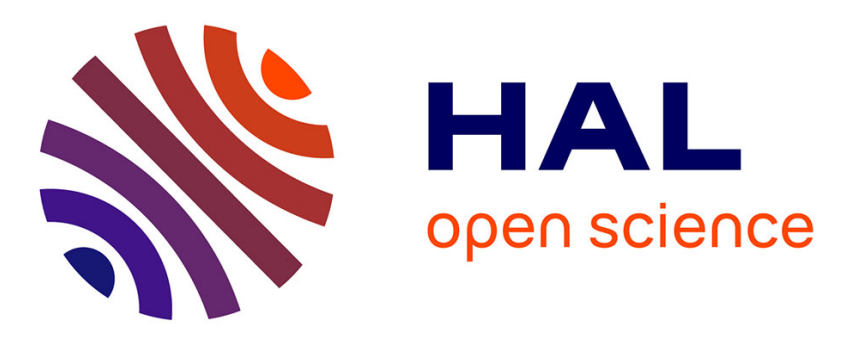

\title{
The ternary system Al-Ni-Ti Part II: Thermodynamic assessment and experimental investigation of polythermal phase equilibria
}

Kejun Zeng, Rainer Schmid-Fetzer, Bertrand Huneau, Peter Rogl, Joseph Bauer

\section{To cite this version:}

Kejun Zeng, Rainer Schmid-Fetzer, Bertrand Huneau, Peter Rogl, Joseph Bauer. The ternary system Al-Ni-Ti Part II: Thermodynamic assessment and experimental investigation of polythermal phase equilibria. Intermetallics, 1999, 7 (12), pp.1347-1359. 10.1016/S0966-9795(99)00055-2 . hal01007378

\section{HAL Id: hal-01007378 \\ https://hal.science/hal-01007378}

Submitted on 7 Oct 2017

HAL is a multi-disciplinary open access archive for the deposit and dissemination of scientific research documents, whether they are published or not. The documents may come from teaching and research institutions in France or abroad, or from public or private research centers.
L'archive ouverte pluridisciplinaire HAL, est destinée au dépôt et à la diffusion de documents scientifiques de niveau recherche, publiés ou non, émanant des établissements d'enseignement et de recherche français ou étrangers, des laboratoires publics ou privés. 


\title{
The ternary system Al-Ni-Ti Part II: Thermodynamic assessment and experimental investigation of polythermal phase equilibria
}

\author{
K. Zeng a , R. Schmid-Fetzer ${ }^{\mathrm{a}, *}, \mathrm{~B}$. Huneau ${ }^{\mathrm{a}, \mathrm{b}, \mathrm{c}}, \mathrm{P}$. Rogl ${ }^{\mathrm{b}}, \mathrm{J}$. Bauer ${ }^{\mathrm{c}}$ \\ a AG Elektronische Materialien, Technische Universität Clausthal, Robert Koch Strasse 42, D-38678 Clausthal-Zellerfeld, Germany \\ ' Institut für Physikalische Chemie der Universität Wien, Währingerstrasse 42, A-1090 Wien, Austria \\ ${ }^{\mathrm{c}}$ Laboratoire de Chimie du Solide et Inorganique Moléculaire, UMR CNRS 6538, Université de Rennes I, Avenue du Général Leclerc, \\ F-35042 Rennes Cedex, France
}

\begin{abstract}
The Al-Ni-Ti phase diagram has been thermodynamically assessed and a consistent set of thermodynamic functions has been developed. The thermodynamic modeling is based on an experimental investigation of the phase equilibria in the composition range of $0.1 \leqslant \mathrm{x}_{\mathrm{A} 1} \leqslant 0.7$. Alloys were prepared by argon-arc or vacuum-electron beam melting of elemental powder blends. X-ray powder diffraction, metallography, SEM and EMPA-techniques were employed to analyze the samples in the as-cast state as well as after annealing at 800,900 and $1000^{\circ} \mathrm{C}$. The existence of the four ternary compounds, $\tau_{1}$ to $\tau_{4}$, has been confirmed, although homogeneity regions differ significantly from reports in the literature. The homogeneous phase, previously claimed at " $\mathrm{Al}_{23} \mathrm{Ni}_{26} \mathrm{Ti}_{51}$ ", is shown by high resolution microprobe and X-ray diffraction measurements to be an extremely fine-grained eutectic structure. The congruent melting behavior of $\tau_{4}=\mathrm{AlNi}_{2} \mathrm{Ti}$ is confirmed, but, in contrast to earlier reports, primary crystallization and congruent melting have been observed for $\tau_{1}=\mathrm{Al}_{13} \mathrm{Ni}_{2} \mathrm{Ti}_{5}$ and $\tau_{3}=\mathrm{Al}_{3} \mathrm{NiTi}_{2}$. In contrast to earlier assessments, $\tau_{1}, \tau_{2}$ and $\tau_{3}$ are experimentally found to be stable at 800,900 and $1000^{\circ} \mathrm{C}$. The thermodynamic modeling of the ternary phases $\tau_{2}$ and $\tau_{3}$ is done with simplified sublattice models, considering their crystal structure and homogeneity ranges. The sublattice model for $\tau_{4}$ is taken from an earlier asessment of the nickel-rich ternary phase equilibria. The present assessment covers the entire composition range. An application to the solidification behavior of ternary alloys is also exemplified.
\end{abstract}

Keywords: A. Aluminides, miscellaneous; A. Laves phases; A. Titanium aluminides, based on $\mathrm{Ti}_{3} \mathrm{Al}$; B. Phase diagram; E. Phase diagram prediction

\section{Introduction}

The thermodynamic description of the $\mathrm{Ti}-\mathrm{Ni}-\mathrm{Al}$ phase diagram was recently optimized by Dupin [1]. Due to the lack of experimental data on the $\mathrm{Ti}-\mathrm{Al}$ side of the phase diagram, however, the work focused on the $\mathrm{Ni}$-rich region with more than $50 \mathrm{at} \% \mathrm{Ni}$. In order to cover the white spot of the system, experiments were designed to provide phase relation data in the Ni-poor region at $900^{\circ} \mathrm{C}$ [2]. The existence of the ternary compounds, $\tau_{1}$ to $\tau_{4}$ (see Table 1 below) was confirmed at that temperature, but, in contrast to earlier investigations and assessments $[3,4,5]$ at significantly different compositions and with different shape of homogeneity regions. This is particularly true for the phase regions of

\footnotetext{
* Corresponding author.
}

$\tau_{3}=\mathrm{Al}_{3} \mathrm{NiTi}_{2}$ with the $\mathrm{MgZn}_{2}$-type structure and for $\tau_{2}=\mathrm{Al}_{2} \mathrm{NiTi}$. The complex atom-site substitution mechanism in $\tau_{3}$, changing from $\mathrm{Ti} / \mathrm{Al}$ exchange at $\mathrm{Al}-$ poor compositions towards $\mathrm{Ni} / \mathrm{Al}$ replacement for the Al-rich part, was monitored in detail by quantitative $\mathrm{X}$ ray powder diffraction techniques (Rietveld analyses) [2].

As part of our systematic study of the phase equilibria and crystal structures of the phases within the quaternary system $\mathrm{Al}-\mathrm{N}-\mathrm{Ni}-\mathrm{Ti}$, the present paper reports a thermodynamic assessment of the Al-Ni-Ti ternary system incorporating the latest experimental results of the primary crystallization behavior of the ternary compounds in the composition range of $0.1 \leqslant \mathrm{x}_{\mathrm{Al}} \leqslant 0.7$, their crystal structures, as well as phase diagram data at 800 and $1000^{\circ} \mathrm{C}$.

The thermodynamic descriptions of the ternary compounds $\tau_{2}=\mathrm{Al}_{2} \mathrm{NiTi}$ and $\tau_{3}=\mathrm{Al}_{3} \mathrm{NiTi}_{2}$ were reoptimized in accordance with the new experimental results. The 
Table 1

Notation of phases in the Al-Ni-Ti system

\begin{tabular}{llll}
\hline Phases & Symbols & Phases & Symbols \\
\hline $\mathrm{NiTi}$ & $\beta_{2}$ & $\mathrm{Al}_{11} \mathrm{Ti}_{5}$ & $\mathrm{Al}_{11} \mathrm{Ti}_{5}$ \\
$\mathrm{Ni}_{3} \mathrm{Ti}$ & $\eta$ & $\mathrm{Al}_{2} \mathrm{Ti}$ & $\mathrm{Al}_{2} \mathrm{Ti}$ \\
$\mathrm{Ni} \mathrm{Ti}{ }_{2}$ & $\delta$ & $\mathrm{Al}_{3} \mathrm{Ti}$ & $\theta$ \\
$\mathrm{AlNi}$ & $\beta_{1}$ & $\mathrm{AlTi}$ & $\kappa$ \\
$\mathrm{AlNi}_{3}$ & $\gamma^{\prime}$ & $\mathrm{AlTi}_{3}$ & $\alpha$ \\
$\mathrm{Al}_{3} \mathrm{Ni}_{2}$ & $v$ & $(\alpha \mathrm{Ti})$ & $(\alpha \mathrm{Ti})$ \\
$\mathrm{Al}_{3} \mathrm{Ni}$ & $\varepsilon$ & $(\beta \mathrm{Ti})$ & $(\beta \mathrm{Ti})$ \\
$\mathrm{Al}_{3} \mathrm{Ni}_{5}$ & $\mathrm{Al} \mathrm{Ni}_{3}$ & $\mathrm{Al}_{13} \mathrm{Ni}_{2} \mathrm{Ti}_{5}$ & $\tau_{1}$ \\
$(\mathrm{Ni})$ & $(\mathrm{Ni})$ & $\mathrm{Al}_{2} \mathrm{NiTi}_{5}$ & $\tau_{2}$ \\
$(\mathrm{Al})$ & $(\mathrm{Al})$ & $\mathrm{Al}_{3} \mathrm{NiTi}_{2}$ & $\tau_{3}$ \\
& & $\mathrm{AlNi}_{2} \mathrm{Ti}$ & $\tau_{4}$ \\
\hline
\end{tabular}

ternary compound richest in $\mathrm{Al}, \tau_{1}=\mathrm{Al}_{13} \mathrm{Ni}_{2} \mathrm{Ti}_{5}$, was included. The thermodynamic descriptions optimized by Dupin [1] of the ternary solution phases and the ternary compound $\tau_{4}=\mathrm{AlNi}_{2} \mathrm{Ti}$ were kept unchanged.

\section{Experimental data}

The literature data on the Al-Ni-Ti system have been critically reviewed in Refs. [3,4] to which readers are referred for details of the previous experimental phase diagrams. Here only a brief discussion of our recent experimental results is presented. Our techniques for sample preparation and heat treatment as well as the methods of characterization by X-ray diffraction (XRD), light optical microscopy (LOM), and electron microprobe analyses (EMPA) have been discussed in detail in our foregoing paper [2] and thus shall not be repeated here. The Greek notations of the phases employed in the review by Budberg [3] will be used throughout the paper (see Table 1).

\subsection{Ternary compounds and liquidus surface}

In the reviews of the $\mathrm{Al}-\mathrm{Ni}-\mathrm{Ti}$ system [3,4], the existence of four ternary compounds was accepted, i.e. $\tau_{1}=\mathrm{Al}_{13} \mathrm{Ni}_{2} \mathrm{Ti}_{5}, \quad \tau_{2}=\mathrm{Al}_{2} \mathrm{NiTi}, \quad \tau_{3}=\mathrm{Al}_{3} \mathrm{NiTi}_{2}, \quad$ and $\tau_{4}=\mathrm{AlNi}_{2} \mathrm{Ti}$. Our recent experiments confirmed the existence of these four phases, but found significantly different composition ranges for $\tau_{2}$ and $\tau_{3}$. According to the new results, $\tau_{3}$ was given a formula $\mathrm{Al}_{3} \mathrm{NiTi}_{2}$ [2].

Crystallographic data from X-ray powder diffraction and the results of the EMPA are summarized in Table 2 for a selection of alloys revealing the presence of the ternary compounds $\tau_{1}, \tau_{2}, \tau_{3}$ and $\tau_{4}$. Agreement exists on the formation of a pronounced field of primary crystallization of the congruent phase $\tau_{4}[3,5]$. The ternary compounds $\tau_{1}$ to $\tau_{3}$ were hitherto, however, described as incongruently melting phases with rather restricted thermal stability ranges [3]. In Dupin's thermodynamic modeling
[1], $\tau_{3}$ was considered as a congruent phase although its liquidus surface was very small and was located in the Ti-corner. Our LOM, SEM and EMPA analysis of alloys prepared at or close to the stoichiometric compositions of these compounds undoubtedly reveal a primary crystallization behavior resulting in congruent melting for $\tau_{1}$ and $\tau_{3}$ (see Figs. 1 and 2). Particularly $\tau_{3}$ precipitated as the primary phase in a large composition area with less than 40 at $\% \mathrm{Ni}$. The evaluation of microstructures as a function of annealing treatment is shown for the $\tau_{4}+\gamma^{\prime}+\eta$ field (Fig. 3) as well as for the fine grained structures $\alpha_{2}+\tau_{3}$ (Figs. 4 and 5).

\subsection{Phase equilibria at 800,900 and $1000^{\circ} \mathrm{C}$}

Phase relations in the ternary system $\mathrm{Al}-\mathrm{Ni}-\mathrm{Ti}$ at $900^{\circ} \mathrm{C}$ were experimentally re-established in detail in the composition range of $0.1 \leqslant \mathrm{x}_{\mathrm{Al}} \leqslant 0.7$ [2]. The results of this experimental investigation employing X-ray powder diffraction, metallography, SEM and EMPA-techniques on about 40 samples were at significant variation to the assessments and investigations hitherto presented $[3,4,5]$. Based on our experiments [2], the conclusions for $900^{\circ} \mathrm{C}$ (see Fig. 6) were as follows: (1) $\tau_{3}$ is not stable below 30 at\% $\mathrm{Al}$ and its banana-shaped homogeneity region bends towards the Al-Ti binary reaching almost 50 at $\% \mathrm{Al} ;$ (2) the low Al-end of $\tau_{2}$ (as shown by Nash [5] actually belongs to $\tau_{3}$; the Al-rich part of $\tau_{2}$ (as suggested by Nash [5]) thus represents the true Al-poor end of $\tau_{2}$ stretching now somewhat to higher Al-concentrations, and (3) there is no two-phase equilibrium $\kappa+\tau_{2}$. The three-phase equilibrium $\alpha_{2}+\kappa+\tau_{3}$ has been recently confirmed at $900^{\circ} \mathrm{C}$ [6]. Heat treatments at 800 and $1000^{\circ} \mathrm{C}$ prove the stability of $\tau_{1}, \tau_{2}$ and $\tau_{3}$ at these temperatures in contrast to earlier assessments [3] (see Table 2 and Fig. 7). Lattice parameter and EMPA data show no significant variations in the extent and size of the homogeneous regions of the ternary compounds at 800,900 or at $1000^{\circ} \mathrm{C}$.

\section{Thermodynamic modeling}

\subsection{Phase stability of pure elements}

The Gibbs energies of pure elements versus temperature $G_{i}^{0}(T)=G_{i}(T)-H_{i}^{S E R}$ are represented by Eq. (1):

$$
\begin{aligned}
G_{i}^{0}(T)= & a+b \mathrm{~T}+c \mathrm{~T} \ln (T)+d \mathrm{~T}^{2}+e \mathrm{~T}^{-1}+f \mathrm{~T}^{3} \\
& +i \mathrm{~T}^{4}+j \mathrm{~T}^{7}+k \mathrm{~T}^{-9}
\end{aligned}
$$

The $G_{i}^{0}(T)$ data are referred to the constant enthalpy value of the so-called Standard Element Reference, $H_{\mathrm{i}}^{\mathrm{SER}}$, at $298.15 \mathrm{~K}$ and 1 bar as recommended by SGTE (Scientific Group Thermodata Europe) [7]. These reference 
Table 2

Crystallographic data and results of EMPA for ternary alloys Al-Ni-Ti

\begin{tabular}{|c|c|c|c|c|c|c|c|c|c|c|c|}
\hline \multirow[t]{2}{*}{$\begin{array}{l}\text { Nominal } \\
\text { composition } \\
\text { in at } \% \mathrm{Al}-\mathrm{Ni}-\mathrm{Ti}\end{array}$} & \multirow[t]{2}{*}{$\begin{array}{l}\text { Heat } \\
\text { treatment } \\
{ }^{\circ} \mathrm{C}\end{array}$} & \multirow[t]{2}{*}{$\begin{array}{l}\text { Phase } \\
\text { analysis }\end{array}$} & \multirow[t]{2}{*}{$\begin{array}{l}\text { Space } \\
\text { group }\end{array}$} & \multirow[t]{2}{*}{$\begin{array}{l}\text { Pearson } \\
\text { symbol }\end{array}$} & \multirow[t]{2}{*}{$\begin{array}{l}\text { Structure } \\
\text { type }\end{array}$} & \multicolumn{2}{|l|}{$\begin{array}{l}\text { Lattice } \\
\text { parameters } \\
\text { pm }\end{array}$} & \multirow{2}{*}{$\begin{array}{l}\text { Volume } \\
10^{6} \mathrm{pm}^{3} \\
\mathrm{~V}\end{array}$} & \multicolumn{3}{|c|}{$\begin{array}{l}\text { Results of } \\
\text { EMPA in } \\
\text { at } \%\end{array}$} \\
\hline & & & & & & a & $\mathrm{c}$ & & $\mathrm{Al}$ & $\mathrm{Ni}$ & $\mathrm{Ti}$ \\
\hline \multirow[t]{4}{*}{$10.7-33.3-56$} & $\operatorname{arc}$ & $\mathrm{NiTi}_{2}$ & $\mathrm{Fd} \overline{3} \mathrm{~m}$ & cF96 & $\mathrm{NiTi}_{2}$ & $1124.82(8)$ & & $1423.1(2)$ & & & \\
\hline & & $\mathrm{NiTi}_{2}$ & $\mathrm{Fd} \overline{3} \mathrm{~m}$ & cF96 & $\mathrm{NiTi}_{2}$ & $1125.30(6)$ & & $1425.0(2)$ & & & \\
\hline & 800 & $\mathrm{AlNi}_{2} \mathrm{Ti}(\tau 4)$ & $\mathrm{Fm} \overline{3} \mathrm{~m}$ & $\mathrm{cF} 16$ & $\mathrm{BiF}_{3}$ & $590.84(4)$ & & $206.26(3)$ & & & \\
\hline & & $\mathrm{NiTi}$ & $\operatorname{Pm} \overline{3} \mathrm{~m}$ & $\mathrm{cP} 2$ & $\mathrm{ClCs}$ & $301.2(1)$ & & $27.32(2)$ & & & \\
\hline \multirow[t]{4}{*}{$14.2-29-56.8$} & $\operatorname{arc}$ & $\mathrm{NiTi}_{2}$ & $\mathrm{Fd} \overline{3} \mathrm{~m}$ & cF96 & $\mathrm{NiTi}_{2}$ & $1124.4(1)$ & & $1421.7(3)$ & & & \\
\hline & & $\mathrm{AlTi}_{3}$ & $\mathrm{P}_{3} / \mathrm{mmc}$ & hP8 & $\mathrm{Ni}_{3} \mathrm{Sn}$ & $578.8(2)$ & $463.9(4)$ & $134.6(1)$ & & & \\
\hline & 800 & $\mathrm{NiTi}_{2}$ & $\mathrm{Fd} \overline{3} \mathrm{~m}$ & cF96 & $\mathrm{NiTi}_{2}$ & $1123.46(6)$ & & $1418.0(1)$ & & & \\
\hline & & $\mathrm{AlTi}_{3}$ & $\mathrm{P}_{3} / \mathrm{mmc}$ & hP8 & $\mathrm{Ni}_{3} \mathrm{Sn}$ & $578.85(1)$ & $463.75(3)$ & $134.56(1)$ & & & \\
\hline \multirow[t]{8}{*}{$14.7-51-34.3$} & arc & $\mathrm{AlNi}_{2} \mathrm{Ti}(\tau 4)$ & $\mathrm{Fm} \overline{3} \mathrm{~m}$ & $\mathrm{cF} 16$ & $\mathrm{BiF}_{3}$ & $591.63(5)$ & & $207.08(3)$ & & & \\
\hline & & NiTi & $\operatorname{Pm} \overline{3} \mathrm{~m}$ & $\mathrm{cP} 2$ & $\mathrm{ClCs}$ & & & & & & \\
\hline & 800 & $\mathrm{AlNi}_{2} \mathrm{Ti}(\tau 4)$ & $\mathrm{Fm} \overline{3} \mathrm{~m}$ & $\mathrm{cF} 16$ & $\mathrm{BiF}_{3}$ & $590.49(9)$ & & $205.89(6)$ & & & \\
\hline & & $\mathrm{NiTi}$ & $\operatorname{Pm} \overline{3} \mathrm{~m}$ & $\mathrm{cP} 2$ & $\mathrm{ClCs}$ & $299.83(2)$ & & $26.95(1)$ & & & \\
\hline & & $\mathrm{Ni}_{3} \mathrm{Ti}$ & $\mathrm{P} 6_{3} / \mathrm{mmc}$ & hP16 & $\mathrm{Ni}_{3} \mathrm{Ti}$ & $511.08(4)$ & $833.5(2)$ & $188.54(4)$ & & & \\
\hline & 1000 & $\mathrm{AlNi}_{2} \mathrm{Ti}(\tau 4)$ & $\mathrm{Fm} 3 \overline{3}$ & cF16 & $\mathrm{BiF}_{3}$ & $590.16(5)$ & & $205.55(3)$ & $19.3(3)$ & $50.9(2)$ & $29.8(3)$ \\
\hline & & $\mathrm{NiTi}$ & $\operatorname{Pm} \overline{3} \mathrm{~m}$ & $\mathrm{cP} 2$ & $\mathrm{ClCs}$ & $297.89(3)$ & & $26.43(1)$ & $6.8(3)$ & $51.8(2)$ & $41.4(3)$ \\
\hline & & $\mathrm{Ni}_{3} \mathrm{Ti}$ & $\mathrm{P} 6_{3} / \mathrm{mmc}$ & hP16 & $\mathrm{Ni}_{3} \mathrm{Ti}$ & $511.22(6)$ & $833.04(9)$ & $188.53(4)$ & & & \\
\hline \multirow[t]{7}{*}{$17.1-43-39.9$} & $\operatorname{arc}$ & $\mathrm{NiTi}_{2}$ & $\mathrm{Fd} \overline{3} \mathrm{~m}$ & cF96 & $\mathrm{NiTi}_{2}$ & $1124.5(1)$ & & $1422.0(3)$ & & & \\
\hline & & $\operatorname{AlNi}_{2} \mathrm{Ti}(\tau 4)$ & $\mathrm{Fm} \overline{3} \mathrm{~m}$ & $\mathrm{cF} 16$ & $\mathrm{BiF}_{3}$ & $591.50(6)$ & & $206.95(4)$ & & & \\
\hline & & NiTi & $\operatorname{Pm} \overline{3} \mathrm{~m}$ & $\mathrm{cP} 2$ & $\mathrm{ClCs}$ & & & & & & \\
\hline & 800 & $\mathrm{NiTi}_{2}$ & $\mathrm{Fd} \overline{3} \mathrm{~m}$ & $\mathrm{cF} 96$ & $\mathrm{NiTi}_{2}$ & $1127.76(8)$ & & $1434.3(2)$ & & & \\
\hline & & $\mathrm{AlNi}_{2} \mathrm{Ti}(\tau 4)$ & $\mathrm{Fm} \overline{3} \mathrm{~m}$ & $\mathrm{cF} 16$ & $\mathrm{BiF}_{3}$ & $590.72(6)$ & & $206.13(4)$ & & & \\
\hline & & NiTi & $\operatorname{Pm} \overline{3} \mathrm{~m}$ & $\mathrm{cP} 2$ & $\mathrm{ClCs}$ & $301.3(1)$ & & $27.36(2)$ & & & \\
\hline & & $\mathrm{Ni}_{3} \mathrm{Ti}$ & $\mathrm{P}_{3} / \mathrm{mmc}$ & hP16 & $\mathrm{Ni}_{3} \mathrm{Ti}$ & $510.51(4)$ & $836.1(2)$ & $188.72(5)$ & & & \\
\hline $20-60-20$ & $\operatorname{arc}$ & $\mathrm{AlNi}_{2} \mathrm{Ti}(\tau 4)$ & $\mathrm{Fm} \overline{3} \mathrm{~m}$ & $\mathrm{cF} 16$ & $\mathrm{BiF}_{3}$ & $586.77(3)$ & & 202.02(2) & & & \\
\hline & & $\mathrm{Ni}_{3} \mathrm{Ti}$ & $\mathrm{P} 6_{3} / \mathrm{mmc}$ & hP16 & $\mathrm{Ni}_{3} \mathrm{Ti}$ & $509.9(1)$ & $831.9(1)$ & 187.34(9) & & & \\
\hline & & $\mathrm{Ni}_{3} \mathrm{Al}$ & $\operatorname{Pm} \overline{3} \mathrm{~m}$ & $\mathrm{cP} 4$ & $\mathrm{AuCu}_{3}$ & $359.49(7)$ & & $46.46(2)$ & & & \\
\hline & 800 & $\mathrm{AlNi}_{2} \mathrm{Ti}(\tau 4)$ & $\mathrm{Fm} \overline{3} \mathrm{~m}$ & $\mathrm{cF} 16$ & $\mathrm{BiF}_{3}$ & $588.12(8)$ & & $203.42(5)$ & & & \\
\hline & & $\mathrm{Ni}_{3} \mathrm{Ti}$ & $\mathrm{P} 6_{3} / \mathrm{mmc}$ & hP16 & $\mathrm{Ni}_{3} \mathrm{Ti}$ & $510.3(1)$ & $831.6(2)$ & $187.53(9)$ & & & \\
\hline & & $\mathrm{Ni}_{3} \mathrm{Al}$ & $\operatorname{Pm} \overline{3} \mathrm{~m}$ & $\mathrm{cP} 4$ & $\mathrm{AuCu}_{3}$ & $359.51(5)$ & & $46.47(1)$ & & & \\
\hline & 1000 & $\mathrm{AlNi}_{2} \mathrm{Ti}(\tau 4)$ & $\mathrm{Fm} \overline{3} \mathrm{~m}$ & cF16 & $\mathrm{BiF}_{3}$ & $586.99(6)$ & & $202.25(4)$ & $22(2)$ & $57(2)$ & $21.5(8)$ \\
\hline & & $\mathrm{Ni}_{3} \mathrm{Ti}$ & $\mathrm{P} 6_{3} / \mathrm{mmc}_{-}$ & hP16 & $\mathrm{Ni}_{3} \mathrm{Ti}$ & $509.95(6)$ & $831.43(7)$ & 187.24(4) & & & \\
\hline & & $\mathrm{Ni} 3 \mathrm{Al}$ & $\operatorname{Pm} 3 \bar{m}$ & $\mathrm{cP} 4$ & $\mathrm{AuCu}_{3}$ & $359.51(5)$ & & $46.47(1)$ & & & \\
\hline $21-30-49$ & arc & $\mathrm{NiTi}_{2}$ & $\mathrm{Fd} \overline{3} \mathrm{~m}$ & cF96 & $\mathrm{NiTi}_{2}$ & $1123.98(7)$ & & $1419.9(2)$ & & & \\
\hline & & $\operatorname{AlNi}_{2} \mathrm{Ti}(\tau 4)$ & $\mathrm{Fm} \overline{3} \mathrm{~m}$ & $\mathrm{cF} 16$ & $\mathrm{BiF}_{3}$ & $590.36(9)$ & & $205.75(6)$ & & & \\
\hline & & $\mathrm{AlTi}_{3}$ & $\mathrm{P} 6_{3} / \mathrm{mmc}$ & hP8 & $\mathrm{Ni}_{3} \mathrm{Sn}$ & $577.1(3)$ & $463.6(6)$ & $113.7(2)$ & & & \\
\hline & & $\operatorname{Ti}(\alpha)$ & $\mathrm{P}_{3} / \mathrm{mmc}$ & $\mathrm{hP} 2$ & $\mathrm{Mg}$ & $289.52(1)$ & $467.34(3)$ & $33.923(3)$ & & & \\
\hline & 800 & $\mathrm{AlTi}_{3}$ & $\mathrm{P}_{3} / \mathrm{mmc}$ & hP8 & $\mathrm{Ni}_{3} \mathrm{Sn}$ & $580.54(5)$ & $465.25(7)$ & $135.79(3)$ & & & \\
\hline & & $\mathrm{AlNi}_{2} \mathrm{Ti}(\tau 4)$ & $\mathrm{Fm} \overline{3} \mathrm{~m}$ & $\mathrm{cF} 16$ & $\mathrm{BiF}_{3}$ & $590.09(10)$ & & $205.47(7)$ & & & \\
\hline & & $\mathrm{NiTi}_{2}$ & $\mathrm{Fd} \overline{3} \mathrm{~m}$ & cF96 & $\mathrm{NiTi}_{2}$ & $1124.6(1)$ & & $1422.4(3)$ & & & \\
\hline $24-20-56$ & $\operatorname{arc}$ & $\mathrm{AlNi}_{2} \mathrm{Ti}(\tau 4)$ & $\mathrm{Fm} \overline{3} \mathrm{~m}$ & $\mathrm{cF} 16$ & $\mathrm{BiF}_{3}$ & $592.4(7)$ & & $207.9(5)$ & & & \\
\hline & & $\mathrm{AlTi}_{3}$ & $\mathrm{P} 6_{3} / \mathrm{mmc}$ & hP8 & $\mathrm{Ni}_{3} \mathrm{Sn}$ & $577.9(5)$ & $465(1)$ & $134.5(4)$ & & & \\
\hline & & $\operatorname{Ti}(\alpha)$ & $\mathrm{P} 6_{3} / \mathrm{mmc}$ & hP2 & $\mathrm{Mg}$ & $289.36(8)$ & $464.9(2)$ & $33.71(2)$ & & & \\
\hline & 800 & $\mathrm{AlTi}_{3}$ & $\mathrm{P} 6_{3} / \mathrm{mmc}$ & hP8 & $\mathrm{Ni}_{3} \mathrm{Sn}$ & $580.57(5)$ & $465.23(7)$ & $135.80(3)$ & & & \\
\hline & & $\mathrm{AlNi}_{2} \mathrm{Ti}(\tau 4)$ & $\mathrm{Fm} \overline{3} \mathrm{~m}$ & $\mathrm{cF} 16$ & $\mathrm{BiF}_{3}$ & $590.04(14)$ & & $205.4(1)$ & & & \\
\hline & & $\mathrm{NiTi}_{2}$ & $\mathrm{Fd} \overline{3} \mathrm{~m}$ & $\mathrm{cF} 96$ & $\mathrm{NiTi}_{2}$ & $1124.77(8)$ & & $1423.0(2)$ & & & \\
\hline $25-50-25$ & arc & $\mathrm{AlNi}_{2} \mathrm{Ti}(\tau 4)$ & $\mathrm{Fm} \overline{3} \mathrm{~m}$ & $\mathrm{cF} 16$ & $\mathrm{BiF}_{3}$ & $589.07(8)$ & & $204.41(6)$ & & & \\
\hline & 800 & $\operatorname{AlNi}_{2} \mathrm{Ti}(\tau 4)$ & $\mathrm{Fm} \overline{3} \mathrm{~m}$ & $\mathrm{cF} 16$ & $\mathrm{BiF}_{3}$ & $589.13(4)$ & & $204.47(1)$ & & & \\
\hline & & $\mathrm{Ni}_{3} \mathrm{Ti}$ & $\mathrm{P} 6_{3} / \mathrm{mmc}$ & hP16 & $\mathrm{Ni}_{3} \mathrm{Ti}$ & $511.6(2)$ & $823(2)$ & $186.6(3)$ & & & \\
\hline & 1000 & $\mathrm{AlNi}_{2} \mathrm{Ti}(\tau 4)$ & $\operatorname{Fm} \overline{3} \mathrm{~m}$ & cF16 & $\mathrm{BiF}_{3}$ & $588.94(5)$ & & $204.27(3)$ & $24.7(1)$ & $50.1(1)$ & $25.1(1)$ \\
\hline & & $\mathrm{Ni}_{3} \mathrm{Ti}$ & $\mathrm{P} 6_{3} / \mathrm{mmc}_{-}$ & hP16 & $\mathrm{Ni}_{3} \mathrm{Ti}$ & $510.88(4)$ & $831.98(8)$ & $188.05(3)$ & & & \\
\hline $27-10-63$ & $\operatorname{arc}$ & $\mathrm{AlNi}_{2} \mathrm{Ti}(\tau 4)$ & $\operatorname{Fm} \overline{3} \mathrm{~m}$ & $\mathrm{cF} 16$ & $\mathrm{BiF}_{3}$ & & & & & & \\
\hline & & $\operatorname{Ti}(\alpha)$ & $\mathrm{P} 6_{3} / \mathrm{mmc}$ & $\mathrm{hP} 2$ & $\mathrm{Mg}$ & $289.62(9)$ & $464.1(3)$ & $33.71(3)$ & & & \\
\hline & 800 & $\mathrm{AlTi}_{3}$ & $\mathrm{P} 6_{3} / \mathrm{mmc}$ & hP8 & $\mathrm{Ni}_{3} \mathrm{Sn}$ & $579.97(5)$ & $464.75(2)$ & $135.38(2)$ & & & \\
\hline & & $\mathrm{AlNi}_{2} \mathrm{Ti}(\tau 4)$ & $\operatorname{Fm} \overline{3} \mathrm{~m}$ & $\mathrm{cF} 16$ & $\mathrm{BiF}_{3}$ & $589.92(8)$ & & $205.30(5)$ & & & \\
\hline & & $\mathrm{NiTi}_{2}$ & $\mathrm{Fd} \overline{3} \mathrm{~m}$ & $\mathrm{cF} 96$ & $\mathrm{NiTi}_{2}$ & $1124.3(2)$ & & $1421.3(5)$ & & & \\
\hline $30-20-50$ & $\operatorname{arc}$ & $\mathrm{Al}_{3} \mathrm{NiTi}_{2}(\tau 3)$ & $\mathrm{P}_{3} / \mathrm{mmc}$ & $\mathrm{hP} 12$ & $\mathrm{MgZn}_{2}$ & $501.20(8)$ & $809.1(2)$ & 176.01(6) & & & \\
\hline & & $\mathrm{AlTi}_{3}$ & $\mathrm{P}_{3} / \mathrm{mmc}$ & hP8 & $\mathrm{Ni}_{3} \mathrm{Sn}$ & $578.3(2)$ & $464.2(2)$ & $134.42(8)$ & & & \\
\hline & 800 & $\mathrm{Al}_{3} \mathrm{NiTi}_{2}(\tau 3)$ & $\mathrm{P}_{3} / \mathrm{mmc}$ & hP12 & $\mathrm{MgZn}_{2}$ & $501.5(3)$ & $805.7(8)$ & $175.5(2)$ & & & \\
\hline
\end{tabular}


Table 2 (continued)

\begin{tabular}{|c|c|c|c|c|c|c|c|c|c|c|c|}
\hline \multirow[t]{2}{*}{$\begin{array}{l}\text { Nominal } \\
\text { composition } \\
\text { in at } \% \text { Al-Ni-Ti }\end{array}$} & \multirow[t]{2}{*}{$\begin{array}{l}\text { Heat } \\
\text { treatment } \\
{ }^{\circ} \mathrm{C}\end{array}$} & \multirow[t]{2}{*}{$\begin{array}{l}\text { Phase } \\
\text { analysis }\end{array}$} & \multirow[t]{2}{*}{$\begin{array}{l}\text { Space } \\
\text { group }\end{array}$} & \multirow[t]{2}{*}{$\begin{array}{l}\text { Pearson } \\
\text { symbol }\end{array}$} & \multirow[t]{2}{*}{$\begin{array}{l}\text { Structure } \\
\text { type }\end{array}$} & \multicolumn{2}{|l|}{$\begin{array}{l}\text { Lattice } \\
\text { parameters } \\
\text { pm }\end{array}$} & \multirow{2}{*}{$\begin{array}{l}\text { Volume } \\
10^{6} \mathrm{pm}^{3} \\
\mathrm{~V}\end{array}$} & \multicolumn{3}{|c|}{$\begin{array}{l}\text { Results of } \\
\text { EMPA in } \\
\text { at } \%\end{array}$} \\
\hline & & & & & & $\mathrm{a}$ & $\mathrm{c}$ & & $\mathrm{Al}$ & $\mathrm{Ni}$ & $\mathrm{Ti}$ \\
\hline & & $\mathrm{AlNi}_{2} \mathrm{Ti}(\tau 4)$ & $\mathrm{Fm} \overline{3} \mathrm{~m}$ & $\mathrm{cF} 16$ & $\mathrm{BiF}_{3}$ & $589.70(4)$ & & $205.06(3)$ & & & \\
\hline & & $\mathrm{AlTi}_{3}$ & $\mathrm{P} 6_{3} / \mathrm{mmc}$ & hP8 & $\mathrm{Ni}_{3} \mathrm{Sn}$ & $579.67(9)$ & $465.10(4)$ & $135.34(4)$ & & & \\
\hline & 1000 & $\mathrm{Al}_{3} \mathrm{NiTi}_{2}(\tau 3)$ & $\mathrm{P}_{3} / \mathrm{mmc}$ & hP12 & $\mathrm{MgZn}_{2}$ & $500.62(2)$ & $807.08(6)$ & $175.16(2)$ & & & \\
\hline & & $\mathrm{AlNi}_{2} \mathrm{Ti}(\tau 4)$ & $\mathrm{Fm} \overline{3} \mathrm{~m}$ & $\mathrm{cF} 16$ & $\mathrm{BiF}_{3}$ & $589.97(3)$ & & $205.35(2)$ & $24.2(2)$ & $49.4(2)$ & $26.4(2)$ \\
\hline & & $\mathrm{AlTi}_{3}$ & $\mathrm{P} 6_{3} / \mathrm{mmc}$ & hP8 & $\mathrm{Ni}_{3} \mathrm{Sn}$ & & & & & & \\
\hline \multirow[t]{6}{*}{$31.3-28.7-40$} & $\operatorname{arc}$ & $\mathrm{AlNi}_{2} \mathrm{Ti}(\tau 4)$ & $\mathrm{Fm} \overline{3} \mathrm{~m}$ & $\mathrm{cF} 16$ & $\mathrm{BiF}_{3}$ & $589.89(5)$ & & $205.26(3)$ & & & \\
\hline & 800 & $\mathrm{Al}_{3} \mathrm{NiTi}_{2}(\tau 3)$ & $\mathrm{P} 6_{3} / \mathrm{mmc}$ & hP12 & $\mathrm{MgZn}_{2}$ & $500.29(7)$ & $806.9(2)$ & $174.90(6)$ & & & \\
\hline & & $\operatorname{AlNi}_{2} \mathrm{Ti}(\tau 4)$ & $\mathrm{Fm} \overline{3} \mathrm{~m}$ & $\mathrm{cF} 16$ & $\mathrm{BiF}_{3}$ & $589.93(7)$ & & $205.30(5)$ & & & \\
\hline & & $\mathrm{AlTi}_{3}$ & $\mathrm{P} 6_{3} / \mathrm{mmc}$ & hP8 & $\mathrm{Ni}_{3} \mathrm{Sn}$ & $580.7(2)$ & $465.1(2)$ & $135.84(8)$ & & & \\
\hline & 1000 & $\mathrm{NiTi}_{2}$ & $\mathrm{Fd} \overline{3} \mathrm{~m}$ & cF96 & $\mathrm{NiTi}_{2}$ & $1124.44(5)$ & & $1421.7(1)$ & & & \\
\hline & & $\mathrm{AlNi}_{2} \mathrm{Ti}(\tau 4)$ & $\mathrm{Fm} \overline{3} \mathrm{~m}$ & $\mathrm{cF} 16$ & $\mathrm{BiF}_{3}$ & $589.92(4)$ & & $205.30(3)$ & $23.8(1)$ & $49.7(1)$ & $26.4(1)$ \\
\hline \multirow[t]{6}{*}{$40-12.5-47.5$} & $\operatorname{arc}$ & $\mathrm{Al}_{3} \mathrm{NiTi}_{2}(\tau 3)$ & $\mathrm{P} 6_{3} / \mathrm{mmc}$ & hP12 & $\mathrm{MgZn}_{2}$ & $502.12(9)$ & $814.1(3)$ & $177.75(8)$ & & & \\
\hline & & $\mathrm{AlTi}_{3}$ & $\mathrm{P} 6_{3} / \mathrm{mmc}$ & hP8 & $\mathrm{Ni}_{3} \mathrm{Sn}$ & $576.8(2)$ & $463.5(2)$ & $133.57(9)$ & & & \\
\hline & 800 & $\mathrm{Al}_{3} \mathrm{NiTi}_{2}(\tau 3)$ & $\mathrm{P} 6_{3} / \mathrm{mmc}$ & hP12hP8 & $\mathrm{MgZn}_{2}$ & $500.93(3)$ & $809.19(8)$ & $175.84(2)$ & $41.5(2)$ & $22.5(4)$ & $36.0(5)$ \\
\hline & & $\mathrm{AlTi}_{3}$ & $\mathrm{P} 6_{3} / \mathrm{mmc}$ & hP8 & $\mathrm{Ni}_{3} \mathrm{Sn}$ & $578.4(1)$ & $464.2(3)$ & $134.49(8)$ & $33.9(6)$ & $1.6(5)$ & $64.5(5)$ \\
\hline & 1000 & $\mathrm{Al}_{3} \mathrm{NiTi}_{2}(\tau 3)$ & $\mathrm{P} 6_{3} / \mathrm{mmc}$ & hP12 & $\mathrm{MgZn}_{2}$ & $501.16(5)$ & $810.4(1)$ & $176.27(3)$ & $41.5(2)$ & $21.6(2)$ & $36.9(2)$ \\
\hline & & $\mathrm{AlTi}_{3}$ & $\mathrm{P} 6_{3} / \mathrm{mmc}$ & hP8 & $\mathrm{Ni}_{3} \mathrm{Sn}$ & $577.51(4)$ & $465.6(3)$ & $134.46(7)$ & $34.8(2)$ & $1.0(4)$ & $64.2(4)$ \\
\hline \multirow[t]{9}{*}{$40-26.6-33.3$} & $\operatorname{arc}$ & $\mathrm{Al}_{3} \mathrm{NiTi}_{2}(\tau 3)$ & $\mathrm{P}_{3} / \mathrm{mmc}$ & hP12 & $\mathrm{MgZn}_{2}$ & $498.78(3)$ & $802.88(7)$ & $172.98(2)$ & & & \\
\hline & & $\mathrm{AlNi}_{2} \mathrm{Ti}(\tau 4)$ & $\mathrm{Fm} \overline{3} \mathrm{~m}$ & $\mathrm{cF} 16$ & $\mathrm{BiF}_{3}$ & $590.1(2)$ & & $205.5(2)$ & & & \\
\hline & & $\mathrm{AlTi}_{3}$ & $\mathrm{P} 6_{3} / \mathrm{mmc}$ & hP8 & $\mathrm{Ni}_{3} \mathrm{Sn}$ & & & & & & \\
\hline & 800 & $\mathrm{Al}_{3} \mathrm{NiTi}_{2}(\tau 3)$ & $\mathrm{P}_{3} / \mathrm{mmc}$ & hP12 & $\mathrm{MgZn}_{2}$ & $499.77(5)$ & $804.01(5)$ & $173.91(3)$ & $37.3(3)$ & $26.4(2)$ & $36.2(3)$ \\
\hline & & $\mathrm{AlNi}_{2} \mathrm{Ti}(\tau 4)$ & $\mathrm{Fm} \overline{3} \mathrm{~m}$ & $\mathrm{cF} 16$ & $\mathrm{BiF}_{3}$ & $589.7(1)$ & & $205.12(9)$ & $25.5(0)$ & $48.6(0)$ & $25.9(0)$ \\
\hline & & $\mathrm{AlTi}_{3}$ & $\mathrm{P} 6_{3} / \mathrm{mmc}$ & hP8 & $\mathrm{Ni}_{3} \mathrm{Sn}$ & & & & & & \\
\hline & 1000 & $\mathrm{Al}_{3} \mathrm{NiTi}_{2}(\tau 3)$ & $\mathrm{P} 6_{3} / \mathrm{mmc}$ & hP12 & $\mathrm{MgZn}_{2}$ & $499.70(2)$ & $803.74(5)$ & $173.80(2)$ & $36.3(1)$ & $26.8(2)$ & $36.9(2)$ \\
\hline & & $\mathrm{AlNi}_{2} \mathrm{Ti}(\tau 4)$ & $\mathrm{Fm} \overline{3} \mathrm{~m}$ & $\mathrm{cF} 16$ & $\mathrm{BiF}_{3}$ & $589.7(2)$ & & $205.1(1)$ & $25.0(3)$ & $48.6(3)$ & $26.3(2)$ \\
\hline & & $\mathrm{AlTi}_{3}$ & $\mathrm{P}_{3} / \mathrm{mmc}$ & hP8 & $\mathrm{Ni}_{3} \mathrm{Sn}$ & & & & & & \\
\hline \multirow[t]{8}{*}{$43-17-40$} & $\operatorname{arc}$ & $\mathrm{Al}_{3} \mathrm{NiTi}_{2}(\tau 3)$ & $\mathrm{P} 6_{3} / \mathrm{mmc}$ & hP12 & $\mathrm{MgZn}_{2}$ & $501.84(3)$ & $816.54(8)$ & $178.08(2)$ & & & \\
\hline & & $\mathrm{AlTi}_{3}$ & $\mathrm{P}_{3} / \mathrm{mmc}$ & hP8 & $\mathrm{Ni}_{3} \mathrm{Sn}$ & $576.25(7)$ & $464.3(1)$ & $133.51(4)$ & & & \\
\hline & 800 & $\mathrm{Al}_{3} \mathrm{NiTi}_{2}(\tau 3)$ & $\mathrm{P} 6_{3} / \mathrm{mmc}$ & hP12 & $\mathrm{MgZn}_{2}$ & $500.91(2)$ & $808.6(1)$ & $175.71(3)$ & $42.7(1)$ & $22.2(3)$ & $35.1(2)$ \\
\hline & & $\mathrm{AlTi}_{3}$ & $\mathrm{P}_{3} / \mathrm{mmc}$ & hP8 & $\mathrm{Ni}_{3} \mathrm{Sn}$ & $578.38(4)$ & $465.1(2)$ & $134.74(6)$ & & & \\
\hline & & $\mathrm{Al}_{2} \operatorname{NiTi}(\tau 2)$ & $\mathrm{Fm} \overline{3} \mathrm{~m}$ & cF116 & $\mathrm{Mn}_{23} \mathrm{Th}_{6}$ & & & & & & \\
\hline & 1000 & $\mathrm{Al}_{3} \mathrm{NiTi}_{2}(\tau 3)$ & $\mathrm{P} 6_{3} / \mathrm{mmc}$ & hP12 & $\mathrm{MgZn}_{2}$ & $501.29(4)$ & $812.86(5)$ & $176.89(3)$ & 43.1(1) & $20.8(1)$ & $36.1(1)$ \\
\hline & & $\mathrm{AlTi}_{3}$ & $\mathrm{P} 6_{3} / \mathrm{mmc}$ & hP8 & $\mathrm{Ni}_{3} \mathrm{Sn}$ & $578.3(1)$ & $465.2(1)$ & $134.72(6)$ & $34.4(5)$ & $2.0(3)$ & $63.6(6)$ \\
\hline & & $(\alpha \mathrm{Ti})$ & $\mathrm{P} 6_{3} / \mathrm{mmc}$ & $\mathrm{hP} 2$ & $\mathrm{Mg}$ & $288.31(2)$ & $4.5768(8)$ & $32.947(6)$ & & & \\
\hline $49.5-23.5-27$ & arc & $\mathrm{Al}_{2} \mathrm{NiTi}(\tau 2)$ & $\mathrm{Fm} \overline{3} \mathrm{~m}$ & cF116 & $\mathrm{Mn}_{23} \mathrm{Th}_{6}$ & $1188.9(1)$ & & $1680.6(4)$ & & & \\
\hline & & $\mathrm{Al}_{3} \mathrm{NiTi}_{2}(\tau 3)$ & $\mathrm{P} 6_{3} / \mathrm{mmc}$ & hP12 & $\mathrm{MgZn}_{2}$ & $501.00(8)$ & $812.3(2)$ & $176.57(6)$ & & & \\
\hline & 800 & $\mathrm{Al}_{2} \mathrm{NiTi}(\tau 2)$ & $\mathrm{Fm} \overline{3} \mathrm{~m}$ & $\mathrm{cF} 116$ & $\mathrm{Mn}_{23} \mathrm{Th}_{6}$ & $1189.49(7)$ & & $1683.0(2)$ & $52.0(3)$ & $24.9(2)$ & $23.1(1)$ \\
\hline & & $\mathrm{Al}_{3} \mathrm{NiTi}_{2}(\tau 3)$ & $\mathrm{P} 6_{3} / \mathrm{mmc}$ & hP12 & $\mathrm{MgZn}_{2}$ & $500.91(2)$ & $808.18(8)$ & $175.61(2)$ & $44.0(4)$ & $22.3(4)$ & $33.7(2)$ \\
\hline & 1000 & $\mathrm{Al}_{2} \mathrm{NiTi}(\tau 2)$ & $\mathrm{Fm} \overline{3} \mathrm{~m}$ & $\mathrm{cF} 116$ & $\mathrm{Mn}_{23} \mathrm{Th}_{6}$ & $1190.68(4)$ & & $1688.1(2)$ & $51.0(1)$ & $24.8(1)$ & $24.2(2)$ \\
\hline & & $\mathrm{Al}_{3} \mathrm{NiTi}_{2}(\tau 3)$ & $\mathrm{P}_{3} / \mathrm{mmc}$ & hP12 & $\mathrm{MgZn}_{2}$ & $500.31(4)$ & $808.7(2)$ & $175.29(4)$ & $43.4(2)$ & $23.0(2)$ & $33.5(1)$ \\
\hline $50-37.5-12.5$ & $\operatorname{arc}$ & $\mathrm{Al}_{2} \mathrm{NiTi}(\tau 2)$ & $\mathrm{Fm} \overline{3} \mathrm{~m}$ & cF116 & $\mathrm{Mn}_{23} \mathrm{Th}_{6}$ & 1188.91(8) & & $1680.6(2)$ & & & \\
\hline & & $\mathrm{AlNi}$ & $\mathrm{Fm} \overline{3} \mathrm{~m}$ & $\mathrm{cP} 2$ & $\mathrm{CsCl}$ & $288.55(5)$ & & & & & \\
\hline & & $\mathrm{Al}_{3} \mathrm{NiTi}_{2}(\tau 3)$ & $\mathrm{P}_{3} / \mathrm{mmc}_{-}$ & hP12 & $\mathrm{MgZn}_{2}$ & $500.03(4)$ & $808.1(1)$ & $174.98(4)$ & & & \\
\hline & 800 & $\mathrm{Al}_{2} \mathrm{NiTi}(\tau 2)$ & $\mathrm{Fm} \overline{3} \mathrm{~m}$ & $\mathrm{cF} 116$ & $\mathrm{Mn}_{23} \mathrm{Th}_{6}$ & $1189.36(7)$ & & $1682.4(2)$ & & & \\
\hline & & $\mathrm{AlNi}$ & $\mathrm{Fm} \overline{3} \mathrm{~m}$ & $\mathrm{cP} 2$ & $\mathrm{CsCl}$ & $289.29(6)$ & & $24.21(1)$ & & & \\
\hline & & $\mathrm{AlNi}_{2} \mathrm{Ti}(\tau 4)$ & $\mathrm{Fm} \overline{3} \mathrm{~m}$ & $\mathrm{cF} 16$ & $\mathrm{BiF}_{3}$ & $588.2(1)$ & & $203.54(7)$ & & & \\
\hline & 1000 & $\mathrm{Al}_{2} \mathrm{NiTi}(\tau 2)$ & $\mathrm{Fm} \overline{3} \mathrm{~m}$ & cF116 & $\mathrm{Mn}_{23} \mathrm{Th}_{6}$ & $1189.62(7)$ & & $1683.5(2)$ & $50.8(1)$ & $25.8(2)$ & $23.7(3)$ \\
\hline & & $\mathrm{AlNi}$ & $\mathrm{Fm} \overline{3} \mathrm{~m}$ & $\mathrm{cP} 2$ & $\mathrm{CsCl}$ & $289.23(4)$ & & $24.195(7)$ & $47.7(2)$ & $49.0(3)$ & $3.3(1)$ \\
\hline & & $\mathrm{AlNi}_{2} \mathrm{Ti}(\tau 4)$ & $\mathrm{Fm} \overline{3} \mathrm{~m}$ & $\mathrm{cF} 16$ & $\mathrm{BiF}_{3}$ & $588.72(6)$ & & $204.05(4)$ & & & \\
\hline $55-10-35$ & $\operatorname{arc}$ & AlTi & $\mathrm{P} 4 / \mathrm{mmm}$ & $\mathrm{tP} 4$ & $\mathrm{AuCu}$ & $399.7(3)$ & $406.2(3)$ & $64.9(1)$ & & & \\
\hline & 800 & AlTi & $\mathrm{P} 4 / \mathrm{mmm}$ & $\mathrm{tP} 4$ & $\mathrm{AuCu}$ & $398.98(6)$ & $408.72(1)$ & $65.06(2)$ & & & \\
\hline & 1000 & AlTi & $\mathrm{P} 4 / \mathrm{mmm}$ & $\mathrm{tP} 4$ & $\mathrm{AuCu}$ & $398.25(4)$ & $407.67(4)$ & $64.66(1)$ & $55.3(5)$ & $0.7(1)$ & $44.1(5)$ \\
\hline & & $\mathrm{Al}_{3} \mathrm{NiTi}_{2}(\tau 3)$ & $\mathrm{P}_{3} / \mathrm{mmc}$ & hP12 & $\mathrm{MgZn}_{2}$ & & & & $49.4(2)$ & $16.1(2)$ & $34.5(1)$ \\
\hline $60-18-22$ & $\operatorname{arc}$ & $\mathrm{Al}_{2} \mathrm{NiTi}(\tau 2)$ & $\mathrm{Fm} \overline{3} \mathrm{~m}$ & cF116 & $\mathrm{Mn}_{23} \mathrm{Th}_{6}$ & $1188.78(7)$ & & $1680.0(2)$ & & & \\
\hline & & $\mathrm{Al}_{13} \mathrm{Ni}_{2} \mathrm{Ti}_{5}(\tau 1)$ & $\mathrm{Pm} \overline{3} \mathrm{~m}$ & $\mathrm{cP} 4$ & $\mathrm{AuCu}_{3}$ & $393.37(7)$ & & $60.87(2)$ & & & \\
\hline & 800 & $\mathrm{Al}_{2} \mathrm{NiTi}(\tau 2)$ & $\mathrm{Fm} \overline{3} \mathrm{~m}$ & cF116 & $\mathrm{Mn}_{23} \mathrm{Th}_{6}$ & $1189.97(8)$ & & $1685.0(3)$ & & & \\
\hline & & $\mathrm{Al}_{13} \mathrm{Ni}_{2} \mathrm{Ti}_{5}(\tau 1)$ & $\operatorname{Pm} \overline{3} \mathrm{~m}$ & $\mathrm{cP} 4$ & $\mathrm{AuCu}_{3}$ & $393.30(7)$ & & $60.84(2)$ & & & \\
\hline & 1000 & $\mathrm{Al}_{2} \mathrm{NiTi}(\tau 2)$ & $\mathrm{Fm} \overline{3} \mathrm{~m}$ & $\mathrm{cF} 116$ & $\mathrm{Mn}_{23} \mathrm{Th}_{6}$ & $1189.14(8)$ & & $1681.5(2)$ & $55.3(2)$ & $23.1(3)$ & $21.4(2)$ \\
\hline & & $\mathrm{Al}_{13} \mathrm{Ni}_{2} \mathrm{Ti}_{5}(\tau 1)$ & $\operatorname{Pm} \overline{3} \mathrm{~m}$ & $\mathrm{cP} 4$ & $\mathrm{AuCu}_{3}$ & $393.27(6)$ & & $60.82(2)$ & $64.0(2)$ & $9.7(1)$ & $26.3(1)$ \\
\hline
\end{tabular}




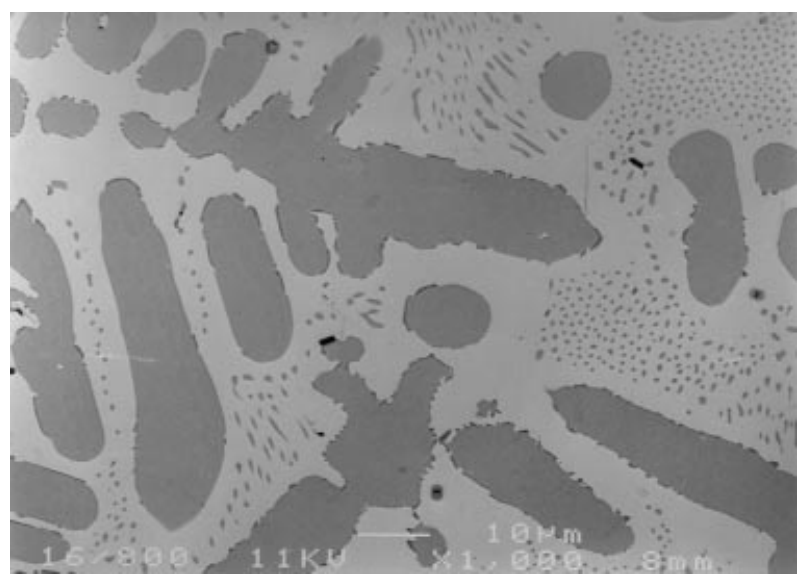

Fig. 1. SEM micrograph of the alloy $60 \mathrm{Al}-18 \mathrm{Ni}-22 \mathrm{Ti}$ (at $\%)$ annealed at $800^{\circ} \mathrm{C}$ and quenched (magnification $1000 \times$ ). The large dark particules are $\tau_{1}=\mathrm{Al}_{13} \mathrm{Ni}_{2} \mathrm{Ti}_{5}\left(\mathrm{Al}_{64} \mathrm{Ni}_{10} \mathrm{Ti}_{26}\right)$ and the white phase is $\tau_{2}=$ $\mathrm{Al}_{2} \mathrm{NiTi}\left(\mathrm{Al}_{55} \mathrm{Ni}_{23.5} \mathrm{Ti}_{21.4}\right)$. Even after annealing the primary dendrites of $\tau_{1}$ can be clearly seen.

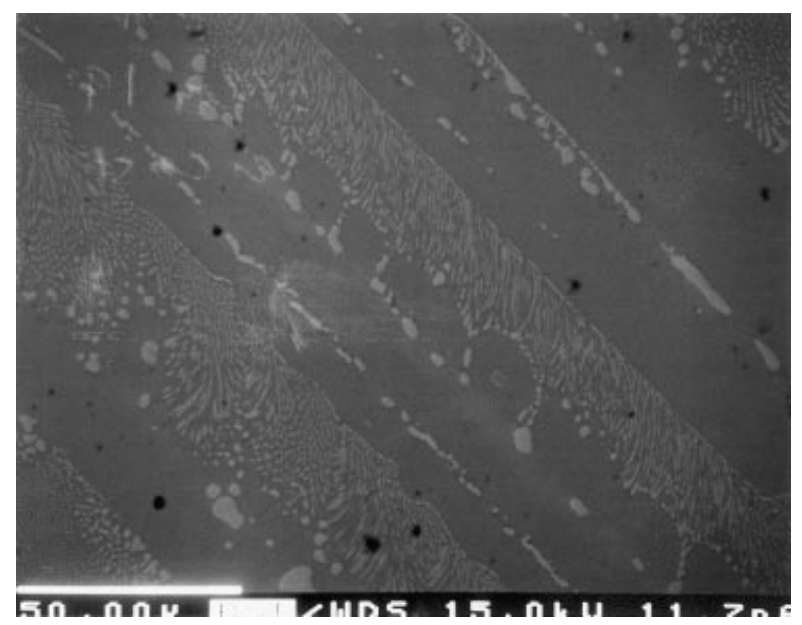

Fig. 2. SEM micrograph of the as-cast alloy $39 \mathrm{Al}-29.5 \mathrm{Ni}-31.5 \mathrm{Ti}$ (at $\%$ ). The black primary phase is $\tau_{3}$ with 27 at $\% \mathrm{Ni}$ and 38.5 at $\% \mathrm{Al}$, and the bright particles are $\tau_{4}$ with 48 at $\% \mathrm{Ni}$ and 24.5 at $\% \mathrm{Al}$.

states are hcp-Ti, fcc-Ni, and fcc-Al. The $G_{i}^{0}(T)$ expressions may be given for several temperature ranges, where the coefficients $a, b, c, d, e, f, i, j$, and $k$ have different values. The $G_{i}^{0}(T)$ functions of the pure elements $\mathrm{Al}, \mathrm{Ni}$ and $\mathrm{Ti}$ were taken from Ref. [7].

\subsection{Summary of modeling of binary boundary systems}

\subsubsection{The Ni-Ti system}

The modeling done by Saunders [8] in the framework of the COST-507 project is used. The phases $\beta_{2}=\mathrm{NiTi}$ and $\eta=\mathrm{Ni}_{3} \mathrm{Ti}$ were described by the two-sublattice model, while $\delta=\mathrm{NiTi}_{2}$ was treated as a line compound. The phase diagram has been well characterized with good overall agreement among the phase diagram studies (Fig. 8). The thermodynamics of the system show some inconsistencies, though.

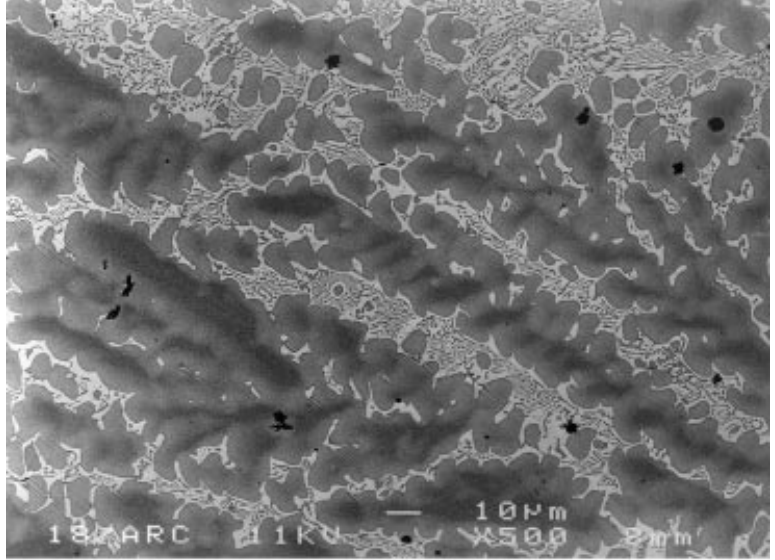

(a)

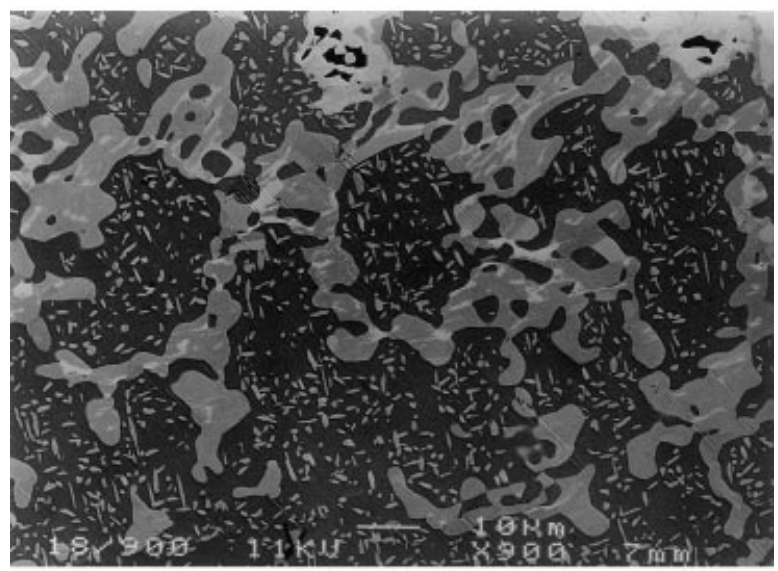

(b)

Fig. 3. SEM micrograph of the as-cast alloy $20 \mathrm{Al}-60 \mathrm{Ni}-20 \mathrm{Ti}$ (at $\%$ ) (magnification $500 \times$ ). The primary dendrites are $\tau_{4}-A l N i_{2} T i$, and the white phase is $\gamma^{\prime}-\mathrm{AlNi}_{3}$. Inset: SEM micrograph of the same alloy after annealing at $800^{\circ} \mathrm{C}$. The black phase is $\tau_{4}-\mathrm{AlNi}_{2} \mathrm{Ti}$, the grey phase is $\gamma^{\prime}-\mathrm{AlNi}_{3}$, and the white particules are $\eta-\mathrm{Ni}_{3} \mathrm{Ti}$.

\subsubsection{The Al-Ti system}

This system was recently modeled four times [9-12]. Since the review of the Al-Ti system by Murray [13], further detailed experimental work has been added. These studies clearly indicated that $(\alpha \mathrm{Ti})$ forms peritectically from the melt and ( $\beta$ Ti) rather than peritectoidally from ( $\beta \mathrm{Ti})$ and $\kappa-\mathrm{AlTi}$ as concluded in Ref. [13]. Based on the experimental results in Refs. [14-16], Saunders [11] took this new feature into account and optimized this system from the then-up-to-date experimental data. The phases and their compositions were based on the experimental data by Kaltenbach et al. [17]. The solid solution phase $(\beta T i)$ was predicted to have a congruent melting point at about 20 at $\%$ Al. Although some important experimental results were published since then, such as the Refs. [18-22] which have been incorporated into the later assessments $[9,10]$ the data by Saunders [11] have been employed in the present work in order to keep consistency 
with the ternary work of Dupin [1] and the COST databank. The calculated phase diagram using Saunders' data is shown in Fig. 9.

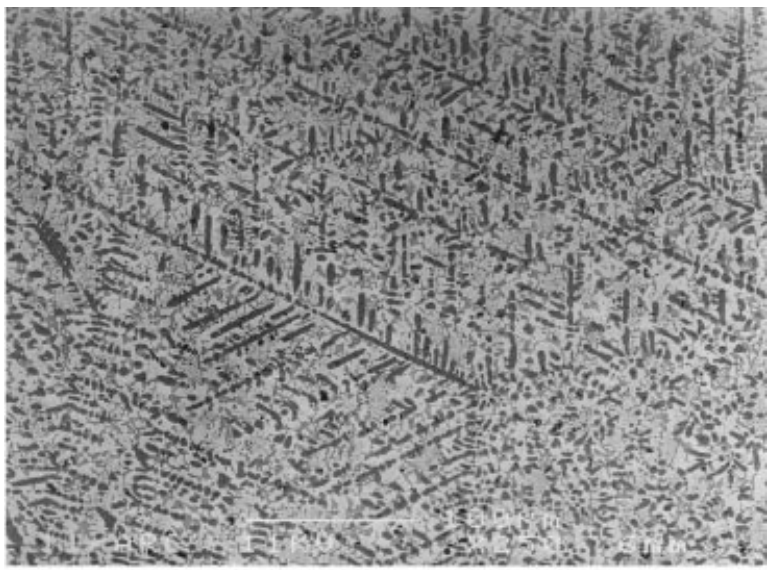

(a)

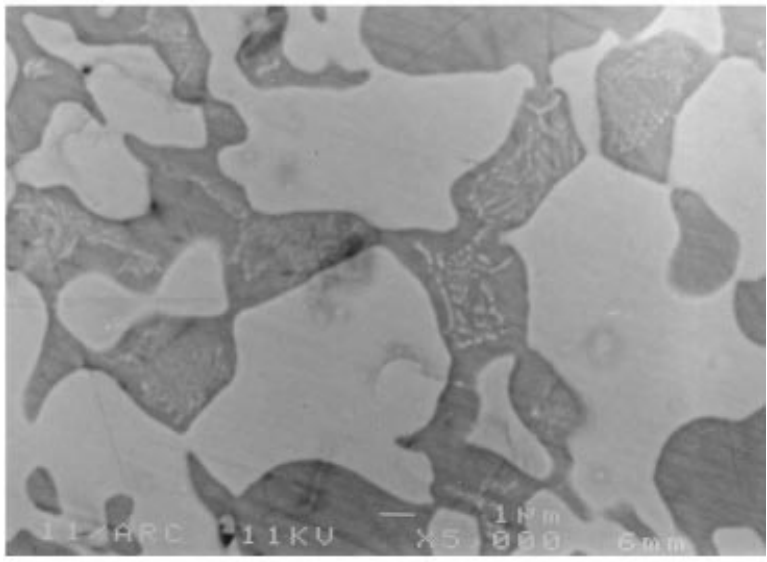

(b)

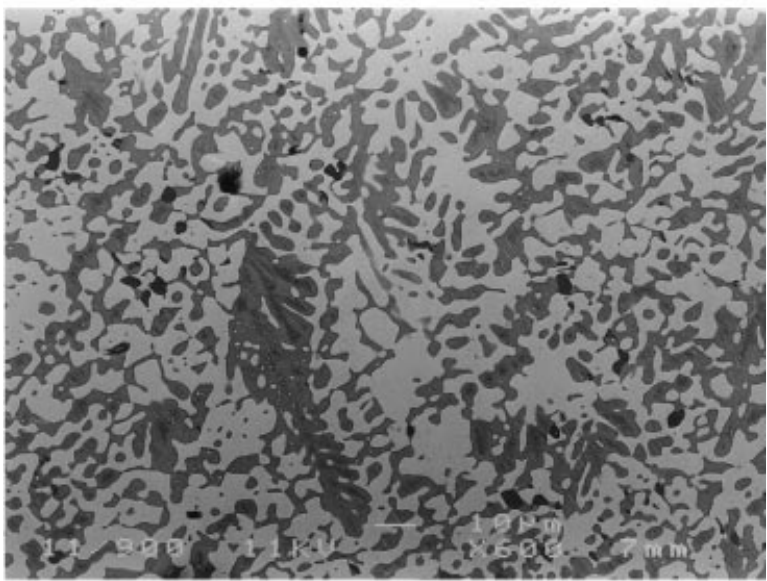

(c)

Fig. 4. SEM micrograph of the alloy $40 \mathrm{Al}-12.5 \mathrm{Ni}-47.5 \mathrm{Ti}$ (at\%), (a) as-cast conditions (magnification $250 \times$ ): the dark dendrites are $\alpha_{2}$ $\mathrm{AlTi}_{3}$ and the white phase is $\tau_{3}$-phase. Inset: a more magnified micrograph of (a) with $5000 \times$. (b) after annealing at $900^{\circ} \mathrm{C}$ (magnification $600 \times)$.

\subsubsection{The Al-Ni system}

The sublattice model was used by Ansara et al. [23] to describe the thermodynamic behaviour of the Al-Ni binary ordered phases $\gamma^{\prime}=\mathrm{AlNi}_{3}, \beta_{1}=\mathrm{AlNi}$, and $v=\mathrm{Al}_{3} \mathrm{Ni}_{2}$. The calculated phase diagram and the thermodynamic

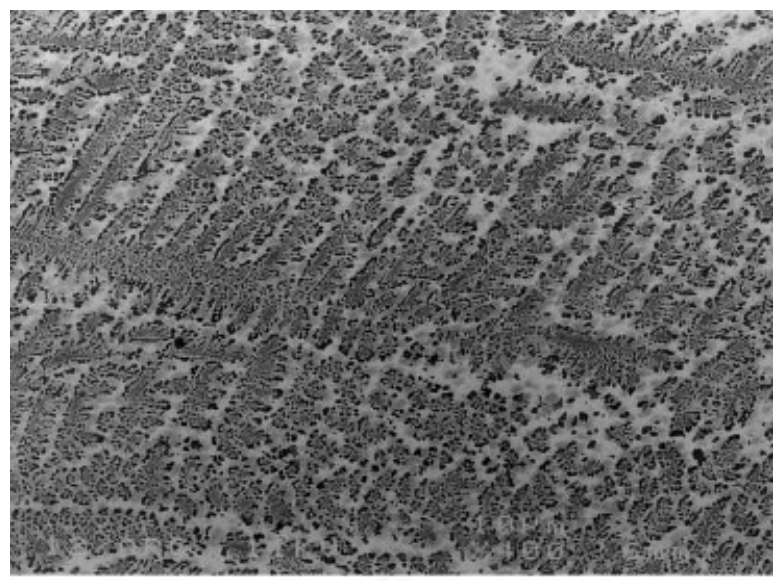

(a)

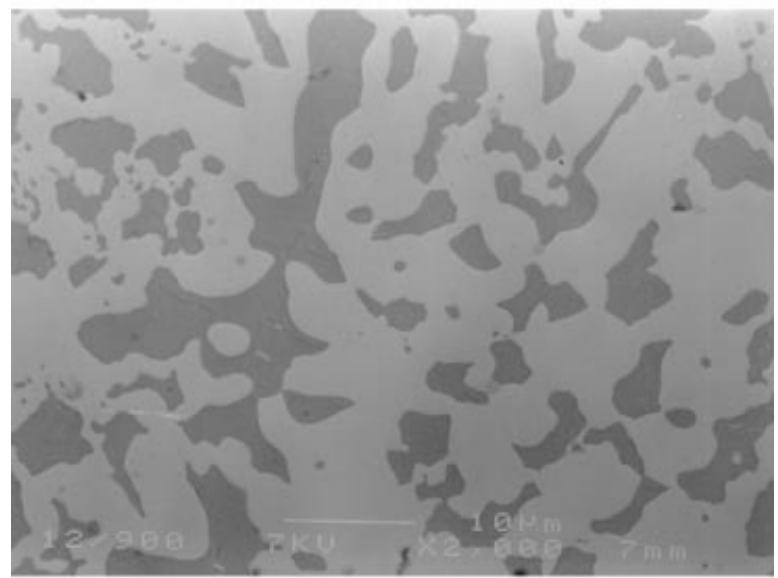

(b)

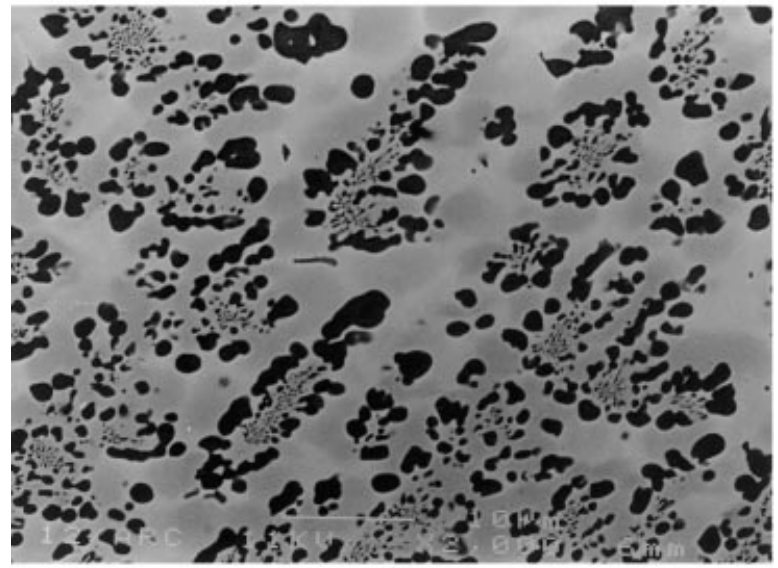

(c)

Fig. 5. SEM micrograph of the alloy $30 \mathrm{Al}-20 \mathrm{Ni}-50 \mathrm{Ti}$ (at $\%$ ), (a) ascast conditions (magnification $400 \times$ ); the dark dendrites are $\tau_{2}-\mathrm{AlTi}_{3}$ and the white phase is $\tau_{3}$-phase. Inset: a more magnified micrograph of (a) with $2000 \times$. (b) after annealing at $900^{\circ} \mathrm{C}$ (magnification $2000 \times$ ). 


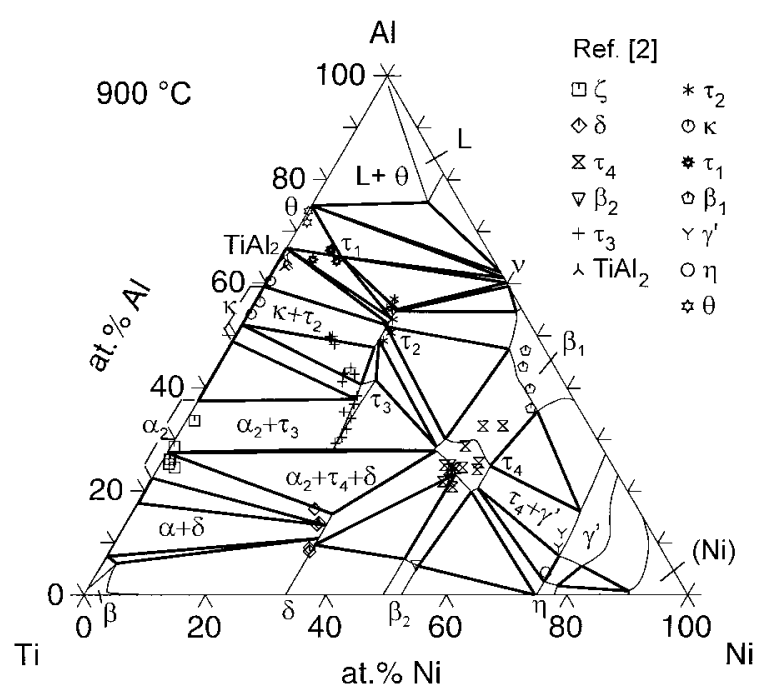

Fig. 6. Calculated isothermal section of the Al-Ni-Ti phase diagram at $900^{\circ} \mathrm{C}$, compared with the experimental data by Huneau et al. [2].

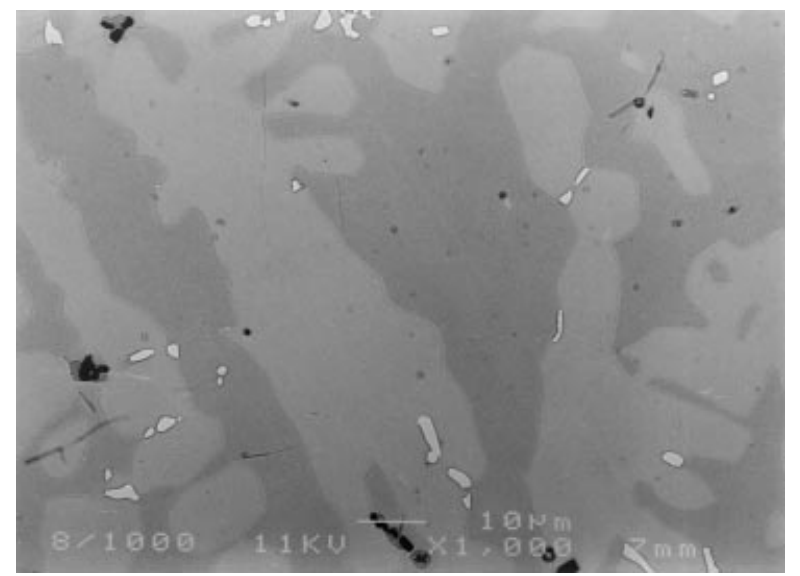

Fig. 7. SEM micrograph of the alloy $49.5 \mathrm{Al}-23.5 \mathrm{Ni}-27 \mathrm{Ti}$ (at $\%$ ) annealed at $1000^{\circ} \mathrm{C}$ (magnification $\left.1000 \times\right)$. The dark grey grains are $\tau_{2}-\mathrm{Al}_{2} \mathrm{NiTi}\left(\mathrm{Al}_{51} \mathrm{Ni}_{25} \mathrm{Ti}_{24}\right)$, the light grey phase is $\tau_{3}$-phase $\left(\mathrm{Al}_{43.5} \mathrm{Ni}_{23} \mathrm{Ti}_{33.5}\right)$, and the white particules are $\tau_{4}-\mathrm{AlNi}_{2} \mathrm{Ti}$.

properties are in very good agreement with the experimental information (Fig. 10).

\subsection{Modeling of ternary phases}

\subsubsection{Solution phases}

The solution phases, $\Phi=$ liquid, fcc, bcc, and hcp, have been described by a substitutional solution model, with a Gibbs energy as follows:

$$
\begin{aligned}
G_{m}^{\Phi}= & x_{\mathrm{Ti}} G_{\mathrm{Ti}}^{0, \Phi}+x_{\mathrm{Ni}} G_{\mathrm{Ni}}^{0, \Phi}+x_{\mathrm{Al}} G_{\mathrm{Al}}^{0, \Phi} \\
& +\mathrm{RT}\left(x_{\mathrm{Ti}} \ln x_{\mathrm{Ti}}+x_{\mathrm{Ni}} \ln x_{\mathrm{Ni}}+x_{\mathrm{Al}} \ln x_{\mathrm{Al}}\right) \\
& +G_{m}^{E, \Phi}
\end{aligned}
$$

where $x_{i}$ is the mole fraction of the element $i, G_{\mathrm{Ti}}^{0, \Phi}, G_{\mathrm{Ni}}^{0, \Phi}$, and $G_{\mathrm{Al}}^{0, \Phi}$ are the molar Gibbs energy of $\mathrm{Ti}, \mathrm{Ni}$, and $\mathrm{Al}$,

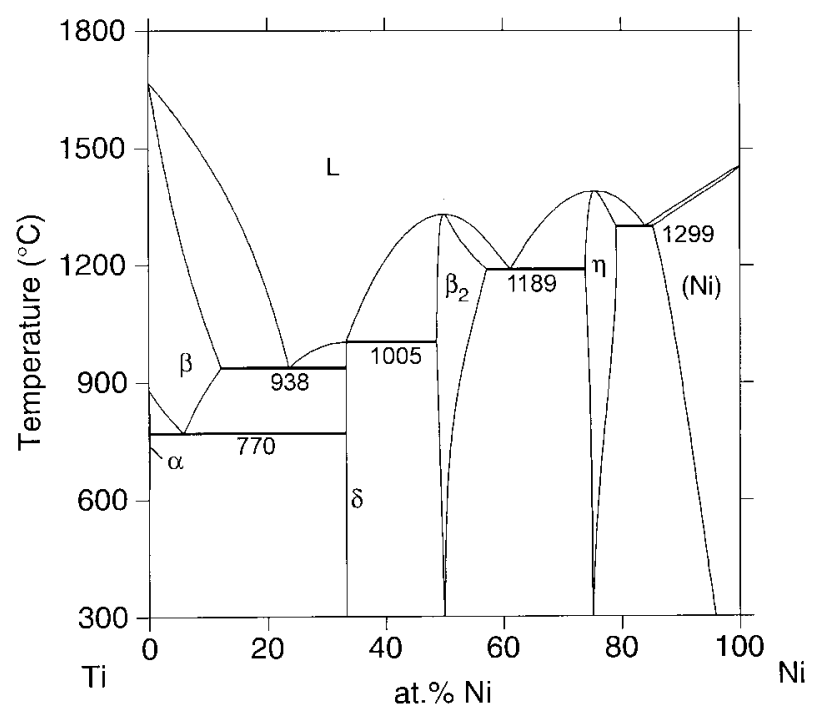

Fig. 8. Calculated Ni-Ti phase diagram using the data of Saunders [8].

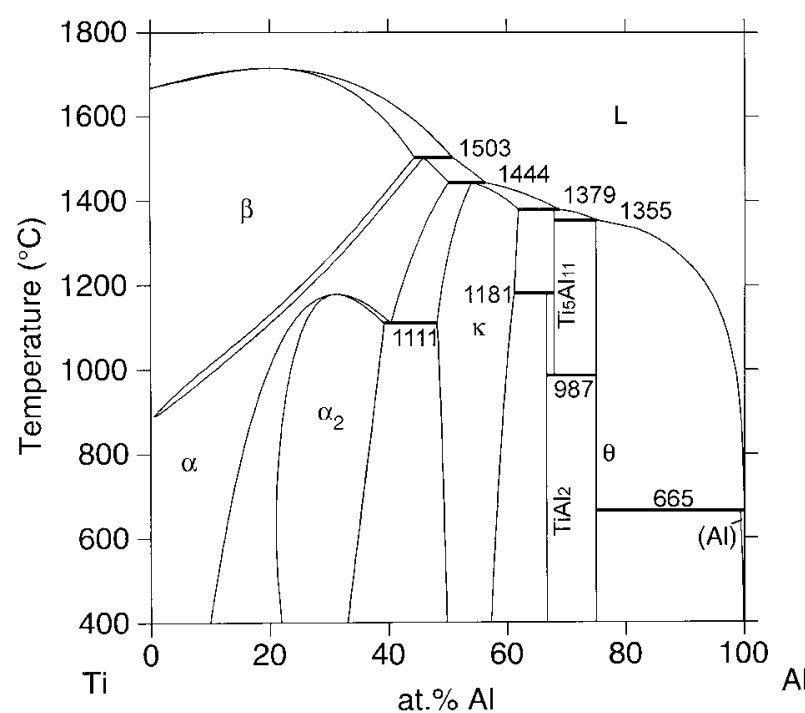

Fig. 9. Calculated Al-Ti phase diagram using the data of Saunders [11].

respectively, with the crystal structure of $\Phi$ and are defined by Eq. (1).

$G_{m}^{E, \Phi}$, the excess Gibbs energy, has been treated as follows:

$$
\begin{aligned}
G_{m}^{E, \Phi}= & x_{\mathrm{Ti}} x_{\mathrm{Ni}} L_{\mathrm{Ti}, \mathrm{Ni}}^{\Phi}+x_{\mathrm{Ti}} x_{\mathrm{Al}} L_{\mathrm{Ti}, \mathrm{Al}}^{\Phi}+x_{\mathrm{Ni}} x_{\mathrm{Al}} L_{\mathrm{Ni}, \mathrm{Al}}^{\Phi} \\
& +x_{\mathrm{Ti}} x_{\mathrm{Ni}} x_{\mathrm{Al}} L_{\mathrm{Ti}, \mathrm{Ni}, \mathrm{Al}}^{\Phi}
\end{aligned}
$$

where $L_{i, j}^{\Phi}$ are binary interaction parameters of the $i-j$ system, which are composition dependent according to the Redlich-Kister formalism [24]:

$L_{i, j}^{\Phi}=\sum_{n=0}^{m} L_{i, j}^{n, \Phi}\left(x_{i}-x_{j}\right)^{n}$ 


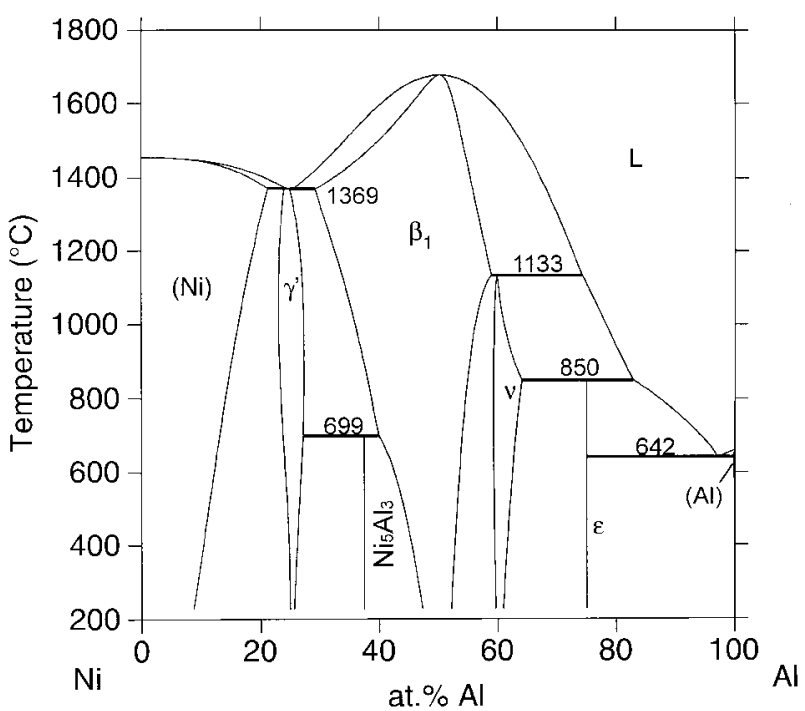

Fig. 10. Calculated Al-Ni phase diagram using the data of Ansara et al. [23].

They have been taken from previously assessed binary systems, i.e. Ni-Ti and $\mathrm{Al}-\mathrm{Ti}$ by Saunders $[8,11]$, and Al-Ni by Ansara et al. [23]. The parameter $L_{\mathrm{Ti}, \mathrm{Ni}, \mathrm{Al}}^{\Phi}$ in Eq. (3) represents the ternary interaction, which is taken to be linearly composition dependent according to the expression:

$L_{\mathrm{Ti}, \mathrm{Ni}, \mathrm{Al}}^{\Phi}=x_{\mathrm{Ti}} L_{\mathrm{Ti}, \mathrm{N}, \mathrm{i}, \mathrm{Al}}^{2,}+x_{\mathrm{Ni}} L_{\mathrm{Ti}, \mathrm{Ni}, \mathrm{Al}}^{1, \Phi}+x_{\mathrm{Al}} L_{\mathrm{Ti}, \mathrm{Ni}, \mathrm{Al}}^{0, \Phi}$

\subsubsection{Ternary compounds}

3.3.2.1. $\tau_{1}\left(A l_{13} \mathrm{Ni}_{2} \mathrm{Ti}_{5}\right)$ and $\tau_{4}\left(\mathrm{AlNi} \mathrm{i}_{2} \mathrm{Ti}\right)$. Although a sublattice model $(\mathrm{Ti}, \mathrm{Ni}, \mathrm{Al})_{3}(\mathrm{Ti}, \mathrm{Ni}, \mathrm{Al})_{1}$ could have been used for $\tau_{1}$ because it essentially is an ordered fcc phase, it was modeled for simplicity as a stoichiometric phase at $\mathrm{Al}_{13} \mathrm{Ni}_{2} \mathrm{Ti}_{5}$. For $\tau_{4}$, the sublattice model employed by Dupin [1] has been adopted.

3.3.2.2. $\tau_{2}\left(A l_{2} N i T i\right)$. Dupin [1] suggested that $\tau_{2}$ was of $\mathrm{Cu}_{16} \mathrm{Mg}_{6} \mathrm{Si}_{7}$-type and, correspondingly, we identified it as of the $\mathrm{Mn}_{23} \mathrm{Th}_{6}$-parent type [2]. Dupin used the sublattice model $(\mathrm{Ti}, \mathrm{Al})_{16}(\mathrm{Ni})_{6}(\mathrm{Ti})_{7}$ to thermodynamically describe this phase as a line compound at 20.69 at $\% \mathrm{Ni}$, about 3.5 at $\%$ Ni less than the homogeneity range measured by us [2].

Model calculations for the crystal structure of $\tau_{2}$ showed general consistency with the structure type of $\mathrm{Mn}_{23} \mathrm{Th}_{6}$ [2]. For comparison, the atom distributions in the sublattices of $\mathrm{Mn}_{23} \mathrm{Th}_{6}$ and $\tau_{2}$ are listed in Table 3 . For practical reasons, the number of sublattices can be decreased by putting the sublattices of the same or similar coordination numbers $(\mathrm{CN})$ with the same occupation of atoms into one sublattice as shown in the last line of Table 3. Thus, we arrive at the sublattice formula $(\mathrm{Al})_{16}(\mathrm{Ti})_{6}(\mathrm{Ni})_{7}$. In order to reproduce the
Table 3

Ideal distributions of atoms on the sublattices of $\mathrm{Mn}_{23} \mathrm{Th}_{6}$ and $\tau_{2}=\mathrm{Al}_{2} \mathrm{NiTi}$

\begin{tabular}{lllllll}
\hline & \multicolumn{6}{l}{ Sublattice with coordination number $\mathrm{CN}$} \\
\cline { 2 - 5 } Phases & $\mathrm{CN} 8$ & $\mathrm{CN} 12$ & $\mathrm{CN} 17$ & $\mathrm{CN} 13$ & $\mathrm{CN} 12$ & Ref. \\
\hline $\mathrm{Mn}_{23} \mathrm{Th}_{6}$ & $4 \mathrm{Mn}$ & $24 \mathrm{Mn}$ & $24 \mathrm{Th}$ & $32 \mathrm{Mn}$ & $32 \mathrm{Mn}$ & Pearson \\
$\tau_{1}=\mathrm{Al}_{2} \mathrm{NiTi}$ & $4 \mathrm{Ni}$ & $24 \mathrm{Ni}$ & $24 \mathrm{Ti}$ & $32 \mathrm{Al}$ & $32 \mathrm{Al}$ & {$[2]$} \\
Sublattice & III & III & II & $\mathrm{I}$ & $\mathrm{I}$ & \\
\hline
\end{tabular}

homogeneity range observed by us [2], Ti must also be introduced into the first sublattice. Although the atom site occupation in $\tau_{2}$ is slightly more complicated [2], the simple formula, $(\mathrm{Ti}, \mathrm{Al})_{16}(\mathrm{Ti})_{6}(\mathrm{Ni})_{7}$ has been employed to model $\tau_{2}$ in the present work. This is still consistent with a line compound but with higher Ni-content than in Dupin's model.

A thermodynamic model for phases with several sublattices was used to describe the Gibbs energy for the individual phases [25]. For one mole of formula unit of the $\tau_{2}$ phase $(\mathrm{Ti}, \mathrm{Al})_{16}(\mathrm{Ti})_{6}(\mathrm{Ni})_{7}$, this model yields the following expression for its Gibbs energy:

$$
\begin{aligned}
G_{m}^{\tau 2}= & y_{\mathrm{Ti}} G_{\mathrm{Ti}: \mathrm{Ti}: \mathrm{Ni}}^{0, \tau 2}+y_{\mathrm{Al}} G_{\mathrm{Al}: \mathrm{Ti}: \mathrm{Ni}}^{0, \tau_{2}} \\
& +16 R T\left(y_{\mathrm{Ti}} \ln y_{\mathrm{Ti}}+y_{\mathrm{Al}} \ln y_{\mathrm{Al}}\right)+G_{m}^{E, \tau 2}
\end{aligned}
$$

The term $y_{i}$ represents the site fraction of component $i$ on the first sublattice it occupies. The colons separate elements on different sublattices. The parameters $G_{\mathrm{Ti}: \mathrm{Ti}: \mathrm{Ni}}^{0, \tau 2}$ and $G_{\mathrm{Al}: \mathrm{Ti}: \mathrm{Ni}}^{0, \tau}$ represent the Gibbs energies of the hypothetical compounds $\mathrm{Ni}_{7} \mathrm{Ti}_{22}$ and $\mathrm{Al}_{16} \mathrm{Ti}_{6} \mathrm{Ni}_{7}$ where the first sublattice is exclusively occupied by $\mathrm{Ti}$ and $\mathrm{Al}$, respectively. They were referred to the standard state SER of the elements (see Section 3.1).

$G_{m}^{E, \tau 2}$ in Eq.(6) is the excess Gibbs energy which is represented by

$G_{m}^{E, \tau 2}=y_{\mathrm{Ti}} y_{\mathrm{Al}} L_{\mathrm{Ti}, \mathrm{Al}: \mathrm{Ti}: \mathrm{Ni}}^{\tau 2}$

where the parameter $L_{\mathrm{Ti}, \mathrm{Al}: \mathrm{Ti}: \mathrm{Ni}}$ represents the interaction energy between $\mathrm{Ti}$ and $\mathrm{Al}$ in the first sublattice, and the comma separates elements that interact on the same sublattice.

3.3.2.3. $\tau_{3}\left(\mathrm{Al}_{3} \mathrm{NiTi}_{2}\right) . \tau_{3}$ was confirmed as of $\mathrm{MgZn}_{2^{-}}$ type in our experiments [2]. The sublattice formula used for this phase by Dupin [1] was $(\mathrm{Ti}, \mathrm{Ni}, \mathrm{Al})_{2}(\mathrm{Ti}, \mathrm{Ni}, \mathrm{Al})_{1}$, but the calculated homogeneity range is almost totally out of the experimental region measured by us [2], showing a much smaller Al-content.

The $\mathrm{MgZn}_{2}$-type Laves structure consists of 3 sublattices with coordination numbers $(\mathrm{CN})$ of 12,12 , and 
16, respectively, containing 12 atoms per unit cell. The larger $\mathrm{Mg}$ atoms occupy the sublattices of $\mathrm{CN} 16$, and the smaller $\mathrm{Zn}$ atoms occupy the sublattices of $\mathrm{CN} 12$ [31]. Consistent with the general conclusion that the percentage of the larger atoms on an atomic site increases with increasing coordination from nearly zero for $\mathrm{CN} 12$ to $100 \%$ for $\mathrm{CN} 16$, we would expect the smaller $\mathrm{Ni}$ as major atom filling the two sublattices of CN12 with 2- and 6-fold lattice sites, and the larger Ti or $\mathrm{Al}$ atoms to fill the 4-fold lattice sites with CN16. Experimental results, however, revealed that $\mathrm{Ni}$ was present in the CN16 sublattice and Al was the major occupier of the CN12 sublattice [2]. In addition, Ti also entered the CN12 sublattice. Therefore, the sublattice formula $(\mathrm{Ti}, \mathrm{Ni}, \mathrm{Al})_{2}(\mathrm{Ti}, \mathrm{Ni})_{1}$ was used in the present work. The difference to Dupin's model is that it is simpler because $\mathrm{Al}$ does not enter the second sublattice.

Similar to Eq. (6), for $1 \mathrm{~mol}$ of formula unit of the $\tau_{3}$ phase $(\mathrm{Ti}, \mathrm{Ni}, \mathrm{Al})_{2}(\mathrm{Ti}, \mathrm{Ni})_{1}$, this model yields the following expression for its Gibbs energy:

$$
\begin{aligned}
G_{m}^{\tau 3}= & y_{\mathrm{Ti}}^{I} y_{\mathrm{Ti}}^{I I} G_{\mathrm{Ti}: \mathrm{Ti}}^{0, \tau 3}+y_{\mathrm{Ti}}^{I} y_{\mathrm{Ni}}^{I I} G_{\mathrm{Ti}}^{0, \tau 3}+y_{\mathrm{Ni}}^{I} y_{\mathrm{Ti}}^{I I} G_{\mathrm{Ni}: \mathrm{Ti}}^{0, \tau 3} \\
& +y_{\mathrm{Ni}}^{I} y_{\mathrm{Ni}}^{I I} G_{\mathrm{Ni}: \mathrm{Ni}}^{0, \tau 3}+y_{\mathrm{Al}}^{I} y_{\mathrm{Ti}}^{I I} G_{\mathrm{Al}: \mathrm{Ti}}^{0, \tau 3}+y_{\mathrm{Al}}^{I} y_{\mathrm{Ni}}^{I I} G_{\mathrm{Al}: \mathrm{Ni}}^{0, \tau 3} \\
& +2 \mathrm{RT}\left(y_{\mathrm{Ti}}^{I} \ln y_{\mathrm{Ti}}^{I}+y_{\mathrm{Ni}}^{I} \ln y_{\mathrm{Ni}}^{I}+y_{\mathrm{Al}}^{I} \ln y_{\mathrm{Al}}^{I}\right) \\
& +\operatorname{RT}\left(y_{\mathrm{Ti}}^{I I} \ln _{\mathrm{Ti}}^{I I}+y_{\mathrm{Ni}}^{I I} \ln y_{\mathrm{Ni}}^{I I}\right)+G_{m}^{E, \tau 3}
\end{aligned}
$$

The term $y_{i}^{n}$ represents the site fraction of component $i$ on the sublattice $n$ it occupies. The parameters $G_{\mathrm{Ti} T \mathrm{Ti}}^{0, \tau 3}$ and $G_{\mathrm{Ni} N \mathrm{Ni}}^{0, \tau 3}$ are the Gibbs energy of the pure components $\mathrm{Ti}$ and $\mathrm{Ni}$, respectively, with the same crystal structure of $\tau_{3}$, or $G_{\mathrm{i}}^{0, \tau 3}(\mathrm{~T})$, as described in Section 3.1; The other $G^{0, \tau 3}$ parameters represent the Gibbs energies of one mole of the hypothetical compounds $\mathrm{NiTi}_{2}, \mathrm{Ni}_{2} \mathrm{Ti}$, $\mathrm{Al}_{2} \mathrm{Ti}$, and $\mathrm{Al}_{2} \mathrm{Ni}$, respectively, formed by the two sublattices with the same crystal structure of $\tau_{3}$.

$G_{m}^{E, \tau 3}$, the excess Gibbs energy, is represented by

$$
\begin{aligned}
G_{m}^{E, \tau 3}= & y_{\mathrm{Ti}}^{I} y_{\mathrm{Ni}}^{I}\left(y_{\mathrm{Ti}}^{I I} L_{\mathrm{Ti}, \mathrm{Ni}: \mathrm{Ti}}^{\tau 3}+y_{\mathrm{Ni}}^{I I} L_{\mathrm{Ti}, \mathrm{Ni} \mathrm{Ni}}^{\tau 3}\right) \\
& +y_{\mathrm{Ni}}^{I} y_{\mathrm{Al}}^{I}\left(y_{\mathrm{Ti}}^{I I} L_{\mathrm{Ni}, \mathrm{Al}: \mathrm{Ti}}^{\tau 3}+y_{\mathrm{Ni}}^{I I} L_{\mathrm{Ni}, \mathrm{Al}: \mathrm{Ni}}^{\tau 3}\right) \\
& +y_{\mathrm{Ti}}^{I} y_{\mathrm{Al}}^{I}\left(y_{\mathrm{Ti}}^{I I} L_{\mathrm{Ti}, \mathrm{Al}: \mathrm{Ti}}^{\tau 3}+y_{\mathrm{Ni}}^{I I} L_{\mathrm{Ti}, \mathrm{Al}: \mathrm{Ni}}^{\tau 3}\right) \\
& +y_{\mathrm{Ti}}^{I I} y_{\mathrm{Ni}}^{I I}\left(y_{\mathrm{Ti}}^{I} L_{\mathrm{Ti}: \mathrm{Ti}, \mathrm{Ni}}^{\tau 3}+y_{\mathrm{Ni}}^{I} L_{\mathrm{Ni}: \mathrm{Ti}, \mathrm{Ni}}^{\tau 3}+y_{\mathrm{Al}}^{I} L_{\mathrm{Al}: \mathrm{Ti}, \mathrm{Ni}}^{\tau 3}\right) \\
& +y_{\mathrm{Ti}}^{I} y_{\mathrm{Ni}}^{I} y_{\mathrm{Al}}^{I}\left(y_{\mathrm{Ti}}^{I I} L_{\mathrm{Ti}, \mathrm{Ni}, \mathrm{Al}: \mathrm{Ti}}^{\tau 3}+y_{\mathrm{Ni}}^{I I} L_{\mathrm{Ti}, \mathrm{Ni}, \mathrm{Al} \mathrm{Ni}}^{\tau 3}\right)
\end{aligned}
$$

The $L^{\tau 3}$ parameters represent the interaction energies between components in the same sublattice dependent of the occupation of the components in the other one. Same to the expression of $\tau_{2}$, the comma here separates elements that interact on the same sublattice, and the colon separate elements on different sublattices.

\section{Results and discussion}

The optimization procedure was started with the $\tau_{3}$ phase at $900^{\circ} \mathrm{C}$. The values of those parameters in Dupin's modeling, which correspond to the same occupations of sublattices, were used as starting values. After the experimental three-phase equilibria $\tau_{3}+\alpha_{2}+$ $\tau_{4}$ and $\delta+\alpha_{2}+\tau_{4}$ were calculated, the parameters of $\tau_{2}$ and $\tau_{1}$ were adjusted successively. Since our work focused on the phase relationships of the ternary compounds $\tau_{1}, \tau_{2}$, and $\tau_{3}$, the following discussion is limited to the region with less than $50 \mathrm{at} \% \mathrm{Ni}$.

\subsection{Isothermal sections}

\subsection{1. $900^{\circ} \mathrm{C}$ section}

The calculated isothermal section at $900^{\circ} \mathrm{C}$ is shown in Fig. 6. A narrow two-phase field $\alpha_{2}+\tau_{4}$ has been calculated, and also the triangle $\alpha_{2}+\tau_{3}+\tau_{4}$ is very narrow. Because the binary Al-Ti parameters account for very well the experimental data in the Ti-rich region of the binary system, the only way to widen this triangle is to modify the $\tau_{4}$ phase so as to reduce the Al-content of the vertex at $\tau_{4}$. But this is outside the scope of the present work because $\tau_{4}$ is the key phase in the Ni-rich region that has been accepted from Dupin [1]. An attempt has been made to increase the Al-content of the lower limit of $\tau_{2}$ and the upper limit of $\tau_{3}$ in Fig. 6 by introducing a less negative interaction parameter between $\mathrm{Al}$ and $\mathrm{Ti}$ in the first sublattice of $\tau_{2}$, i.e. $L_{\mathrm{Ti}, \mathrm{Al}: \mathrm{Ti}: \mathrm{Ni}}$ in Eq. (7). It was successful not only for this purpose, but also to obtain the $\alpha_{2}+\kappa+\tau_{3}$ equilibrium stable at $900^{\circ} \mathrm{C}$ which has been experimentally observed by Bauer et al. [6]. Although at that time only the region close to the $\alpha_{2}+\kappa$ binary was investigated [6], small precipitates $\mathrm{Al}_{40} \mathrm{Ni}_{20} \mathrm{Ti}_{40}$ were identified in equilibrium with $\alpha_{2}+\kappa$, which at that time were speculated to be the " $\tau_{2}-\mathrm{Al}_{2} \mathrm{NiTi}$ " phase cited by Budberg [3] and Nash [5]. From our subsequent reinvestigation of the isothermal section [2], however, it became obvious, that these precipitates crystallographically correspond to $\tau_{3}$ rather than to $\tau_{2}$.

In our experiments, $\tau_{3}$ was found to coexist with $\mathrm{Al}_{2} \mathrm{Ti}$ [2]. This result has not been confirmed by the calculations which gave the competing equilibrium $\kappa(\mathrm{AlTi})+\tau_{2}$ and the adjoining equilibria $\kappa(\mathrm{AlTi})+\tau_{3}$ and $\mathrm{Al}_{2} \mathrm{Ti}+\tau_{2}$ instead. This may be due to an incorrect account of the substitution of $\mathrm{Ti} / \mathrm{Al}$ by $\mathrm{Ni} / \mathrm{Al}$ in $\tau_{3}$. In order to come closer to experimental data either $\tau_{3}$ has to be rendered more stable or $\tau_{2}$ less stable. There is, however, not much room for changes of the relative stabilities owing to the constraints imposed by the other 
surrounding equilibria e.g. $\alpha_{2}+\tau_{4}$. Further experimental and theoretical work is needed to solve this problem.

\subsection{2. $1200^{\circ} \mathrm{C}$ section}

With Dupin's parameters of the liquid phase as well as our parameters of $\tau_{1}, \tau_{2}$, and $\tau_{3}$, the liquid phase was calculated to be stable at $1200^{\circ} \mathrm{C}$ in the $\mathrm{Ni}$-poor region around 15 at $\%$ Ni and $30-55$ at $\%$ Al. The experiments by Mazdiyasni et al. [26] did not find evidence for liquid phase in the alloy with $7.4 \mathrm{at} \% \mathrm{Ni}$ and 64 at\% $\mathrm{Al}$ annealed at this temperature for $500 \mathrm{~h}$. Thus, $L_{\mathrm{Ti}, \mathrm{Ni}, \mathrm{Al}}^{0, L}$ and $L_{\mathrm{Ti}, \mathrm{Ni} A \mathrm{Al}}^{2, L}$ in Eq. (5), which represent the contributions of $\mathrm{Al}$ and $\mathrm{Ti}$, respectively, to the interaction energy in liquid, were changed from Dupin's modeling to prevent it from appearing in this region.

The resulting isothermal section at $1200^{\circ} \mathrm{C}$ is presented in Fig. 11. Five of the six phase equilibria suggested by Mazdiyasni et al. [26] were obtained (see the phase fields marked with star), while the other one, the $\tau_{1}+\tau_{3}$ equilibrium, were not obtained. The $\tau_{3}$ phase was identified by EMPA to be at 49Al-17Ni-34Ti (at \%) in Ref. [26], but its crystal structure was not analyzed by means of X-ray diffraction techniques. Thus, no further attempt has been made to fit this data.

\subsection{3. $800^{\circ} \mathrm{C}$ section}

The calculated phase relationships in the Al-corner at $800^{\circ} \mathrm{C}$ agree excellently with the experimental results by Omarov et al. [27] (see the phase fields marked with star in Fig. 12). In contrast to Dupin's modeling [1], the $\tau_{3}$ phase is predicted unstable at $800^{\circ} \mathrm{C}$, which contradicts the experimental results $[28,29]$. We also observed $\tau_{3}$ at $800^{\circ} \mathrm{C}$, see Table 2 , and no significant change in its phase amount was seen within 10 days. Long time heat

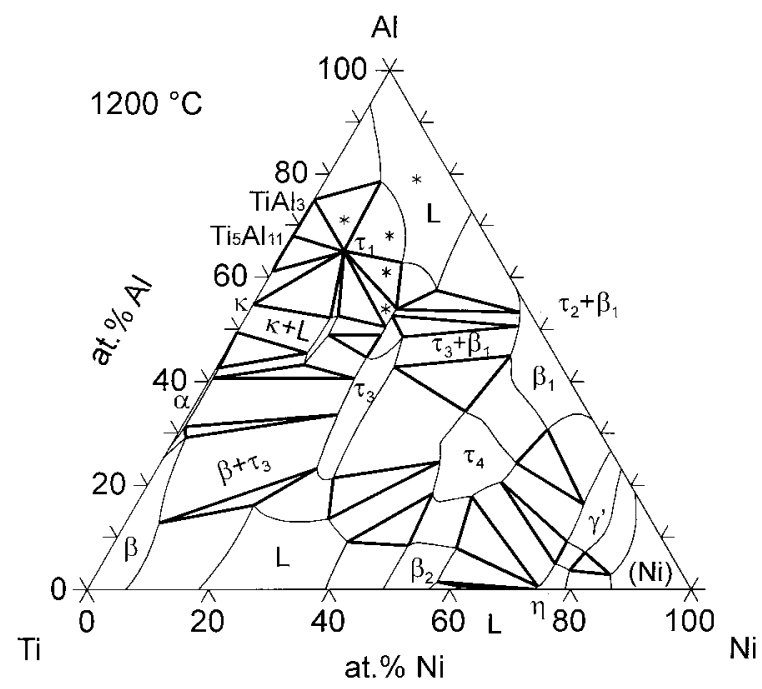

Fig. 11. Calculated isothermal section of the Al-Ni-Ti phase diagram at $1200^{\circ} \mathrm{C}$. The phase fields marked with star "** were suggested by Mazdiyasni et al. [26]. treatments to be sure of its stability have not yet been done. The calculated metastability at $800^{\circ} \mathrm{C}$ is because $\tau_{3}$ has been made less stable at $900^{\circ} \mathrm{C}$ in order to have a stable $\alpha_{2}+\tau_{4}$ tieline. In order to get $\tau_{3}$ at $800^{\circ} \mathrm{C}$, $L_{\mathrm{Ti}, \mathrm{Al}: \mathrm{Ni}}^{\tau 3}$ in Eq. (9) must be more negative than at $900^{\circ} \mathrm{C}$, or $-274606+50 \mathrm{~T}(\mathrm{~J} / \mathrm{mole})$ considering the requirement of the phase equilibria at both temperatures. But with these coefficients, the calculated liquidus surface of $\tau_{3}$ shrinks to the Ni-Ti side by about $50 \%$, and the growing liquidus surface of $\tau_{2}$ would cover most of the experimental data points for the primary phase $\tau_{3}$. The other equilibria determined in [28,29], which do not involve $\tau_{3}$, have been calculated (see the phase fields marked with circle and triangle, respectively, in Fig. 12).

\subsection{Liquidus surfaces}

The experimental data on the primary phases in different alloys are compared with the calculated projection of the liquidus surfaces in Fig. 13(a). Experimental results and calculations are in good agreement. As to those by Nash and Liang [5], however, the two alloys for $\tau_{2}$ and three of the alloys for $\tau_{4}$ were calculated to have $\tau_{3}$ as the primary phase.

The calculated invariant reactions of the system related to the liquidus surfaces are summarized in Table 4 and Fig. 13(b).

\section{Simulation of solidification processes}

The present thermodynamic description of the Al-NiTi system can be applied to simulate the solidification process of ternary alloys. As an example, the solidification

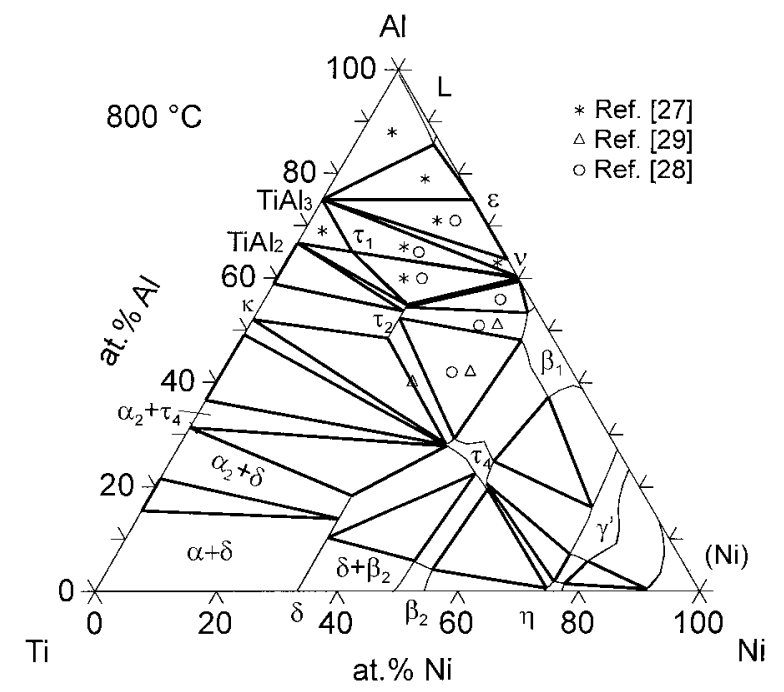

Fig. 12. Calculated isothermal section of the Al-Ni-Ti phase diagram at $800^{\circ} \mathrm{C}$. The phase fields marked with symbols were experimentally observed. 

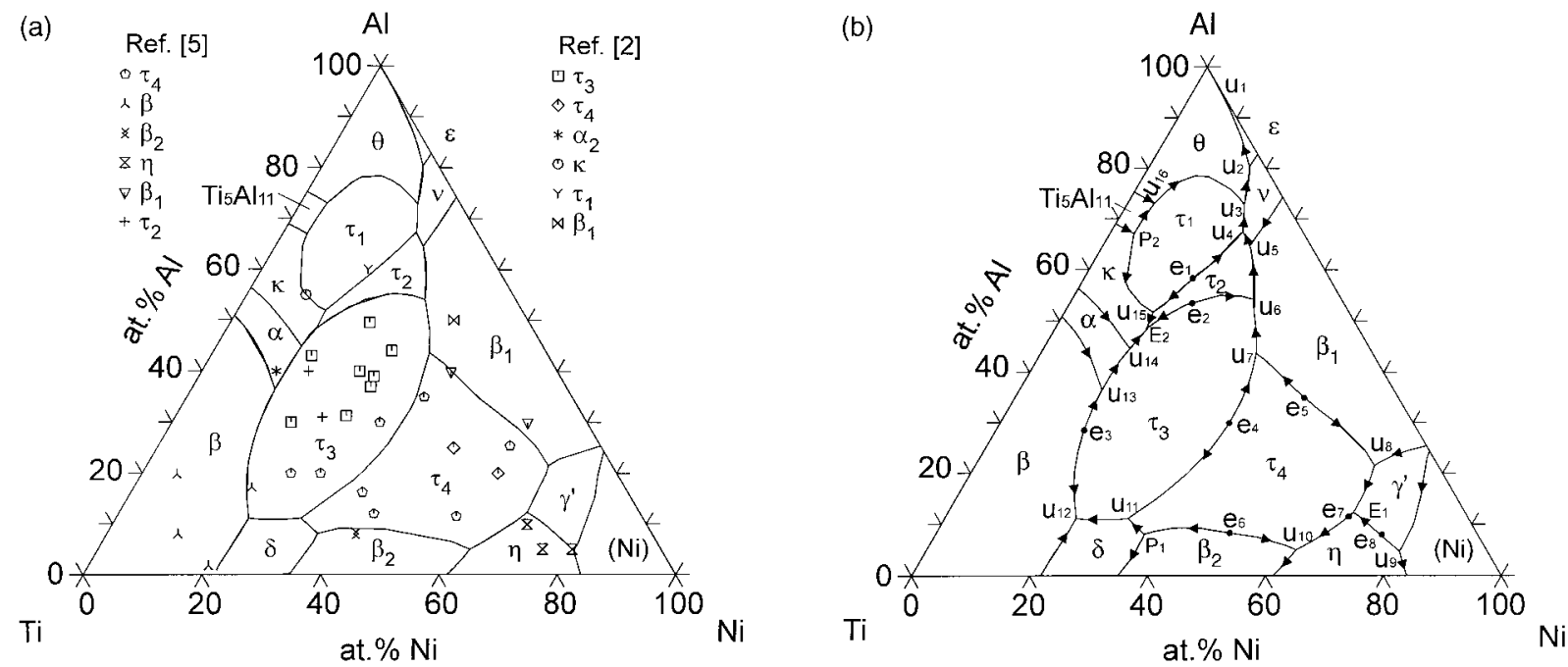

Fig. 13. Projection of the calculated liquidus surfaces. (a) Compared with the alloys whose primary phases have been experimentally determined (b) with the invariant equilibria which involve the liquid phase (see Table 4).

Table 4

Calculated invariant reactions of the $\mathrm{Ti}-\mathrm{Ni}-\mathrm{Al}$ system related to the liquidus surfaces

\begin{tabular}{|c|c|c|c|c|}
\hline \multirow[t]{2}{*}{ Types } & \multirow[t]{2}{*}{ Reactions } & \multicolumn{2}{|c|}{ Liquid compositions } & \multirow[t]{2}{*}{ Temperature $(\mathrm{C})$} \\
\hline & & at $\% \mathrm{Ni}$ & at $\% \mathrm{Al}$ & \\
\hline $\mathrm{U}_{1}$ & $\mathrm{~L}+\theta \leftrightarrow \varepsilon+(\mathrm{Al})$ & 3.06 & 96.85 & 644.7 \\
\hline $\mathrm{U}_{2}$ & $\mathrm{~L}+v \leftrightarrow \varepsilon+\theta$ & 16.96 & 80.41 & 838.8 \\
\hline $\mathrm{U}_{3}$ & $\mathrm{~L}+\tau_{1} \leftrightarrow \theta+v$ & 19.86 & 72.89 & 924 \\
\hline $\mathrm{U}_{4}$ & $\mathrm{~L}+\tau_{2} \leftrightarrow v+\tau_{1}$ & 22.41 & 67.34 & 995.7 \\
\hline $\mathrm{U}_{5}$ & $\mathrm{~L}+\beta_{1} \leftrightarrow \nu+\tau_{2}$ & 24.93 & 64.62 & 1068.3 \\
\hline $\mathrm{U}_{6}$ & $\mathrm{~L}+\tau_{3} \leftrightarrow \beta_{1}+\tau_{2}$ & 30.51 & 54.09 & 1227 \\
\hline $\mathrm{U}_{\underline{7}}$ & $\mathrm{~L}+\tau_{4} \leftrightarrow \tau_{3}+\beta_{1}$ & 36.58 & 43.71 & 1334.8 \\
\hline$\overline{\mathrm{U}_{8}}$ & $\mathrm{~L}+\beta_{1} \leftrightarrow \gamma^{\prime}+\tau_{4}$ & 67.72 & 21.47 & 1312.7 \\
\hline $\mathrm{E}_{1}$ & $\mathrm{~L} \leftrightarrow \gamma^{\prime}+\eta+\tau_{4}$ & 68.77 & 12.27 & 1310.7 \\
\hline $\mathrm{U}_{9}$ & $\mathrm{~L}+\gamma^{\prime} \leftrightarrow(\mathrm{Ni})+\eta$ & 80.38 & 4.80 & 1335 \\
\hline $\mathrm{U}_{10}$ & $\mathrm{~L}+\tau_{4} \leftrightarrow \beta_{2}+\eta$ & 62.71 & 5.03 & 1236.5 \\
\hline $\mathrm{P}_{1}$ & $\mathrm{~L}+\beta_{2}+\tau_{4} \leftrightarrow \delta$ & 35.44 & 8.13 & 1116.4 \\
\hline $\mathrm{U}_{11}$ & $\mathrm{~L}+\tau_{4} \leftrightarrow \delta+\tau_{3}$ & 31.11 & 11.11 & 1110.5 \\
\hline $\mathrm{U}_{12}$ & $\mathrm{~L}+\tau_{3} \leftrightarrow(\beta \mathrm{Ti})+\delta$ & 22.30 & 11.17 & 1063.8 \\
\hline $\mathrm{U}_{13}$ & $\mathrm{~L}+(\beta \mathrm{Ti}) \leftrightarrow(\alpha \mathrm{Ti})+\tau_{3}$ & 13.99 & 36.51 & 1259.9 \\
\hline $\mathrm{U}_{14}$ & $\mathrm{~L}+(\alpha \mathrm{Ti}) \leftrightarrow \tau_{3}+\kappa$ & 14.34 & 44.82 & 1178.6 \\
\hline $\mathrm{U}_{15}$ & $\mathrm{~L}+\tau_{1} \leftrightarrow \tau_{2}+\kappa$ & 14.84 & 51.91 & 1179.5 \\
\hline $\mathrm{E}_{2}$ & $\mathrm{~L} \leftrightarrow \kappa+\tau_{2}+\tau_{3}$ & 15.05 & 48.31 & 1170.6 \\
\hline $\mathrm{P}_{2}$ & $\mathrm{~L}+\mathrm{Al}_{11} \mathrm{Ti}_{5}+\kappa \leftrightarrow \tau_{1}$ & 4.12 & 67.02 & 1337.3 \\
\hline $\mathrm{U}_{16}$ & $\mathrm{~L}+\mathrm{Al}_{11} \mathrm{Ti}_{5} \leftrightarrow \tau_{1}+\theta$ & 4.48 & 73.01 & 1310.4 \\
\hline $\mathrm{e}_{1}$ & & 17.6 & 56.85 & 1270.7 \\
\hline$e_{2}$ & & 20.42 & 54.04 & 1376.7 \\
\hline $\mathrm{e}_{3}$ & & 15 & 28.5 & 1313 \\
\hline $\mathrm{e}_{4}$ & & 38.89 & 29.96 & 1446.2 \\
\hline $\mathrm{e}_{5}$ & & 49.1 & 34.96 & 1482 \\
\hline $\mathrm{e}_{6}$ & & 49.8 & 8.35 & 1342 \\
\hline $\mathrm{e}_{7}$ & & 68.4 & 11.5 & 1310.8 \\
\hline$e_{8}$ & & 75.8 & 8.01 & 1352 \\
\hline
\end{tabular}

of the experimental alloy $39 \mathrm{Al}-29.5 \mathrm{Ni}-31.5 \mathrm{Ti}($ at $\%)$ has been simulated under two extreme conditions: (1) back diffusion in the solid phases is complete (global equilibrium) and (2) no back diffusion in the solids (Scheil model). In both cases a uniform liquid composition and local equilibrium at the solid-liquid interface has been assumed [30]. The calculated amount of the liquid phase varying with temperature during solidification is shown in Fig. 14. The solidification starts at the liquidus point $1896^{\circ} \mathrm{C}$. Under equilibrium condition, the alloy is solidified as a single phase $\tau_{3}$, which means that only the primary solidification step occurs: $L \rightarrow \tau_{3}$. The liquid is consumed at $1530^{\circ} \mathrm{C}$.

Under Scheil conditions, however, the contents of nickel and aluminum in liquid increase further with decreasing temperature and the solidification does not stop at $1530^{\circ} \mathrm{C}$. After the primary crystallization of $\tau_{3}$, the composition path of the liquid phase reaches the valley line of the eutectic reaction $L \rightarrow \tau_{3}+\tau_{4}$ at $1388^{\circ} \mathrm{C}$ (see Fig. 15). This three-phase reaction proceeds down to $1335^{\circ} \mathrm{C}$, where the liquid survives the fourphase equilibrium $L+\tau_{4} \rightarrow \tau_{3}+\beta_{1}\left(\mathrm{U}_{7}\right.$ in Fig. 15) and goes further all the way down until beyond $U_{2}$ in Fig. 13b. At $1230^{\circ} \mathrm{C}$ after $\mathrm{U}_{7}$, however, the amount of residual liquid becomes virtually zero $(0.1 \mathrm{~mol} \%)$. Hence, the whole solidification process practically ends at $1230^{\circ} \mathrm{C}$ before reaching $\mathrm{U}_{6}$. The amounts of liquid consumed in these four steps are 82.7, 10.8, 0.5, and $6.0(\mathrm{~mol} \%)$, successively. Under exact Scheil-conditions (step size $<<1 \mathrm{~K}$ ) the amount of liquid consumed in the transition type reaction $\mathrm{U}_{7}(0.5 \mathrm{~mol} \%)$ should even go to zero. As a result of the blocked solid state diffusion, in addition to the primary $\tau_{3}$ and secondary eutectic $\tau_{3}+\tau_{4}$, there is some eutectic $\tau_{3}+\beta_{1}$ in the solidified microstructures.

The microstructures of the as-cast alloy of 39Al$29.5 \mathrm{Ni}-\mathrm{Ti}(\mathrm{at} \%)$ are presented in Fig. 2. Although the solidification structures in Fig. 2 are not evenly distributed, it can still be seen that the black primary $\tau_{3}$ dominates the picture. There are very fine eutectic structures of $\tau_{3}+\tau_{4}$ in the micrograph, confirming the 


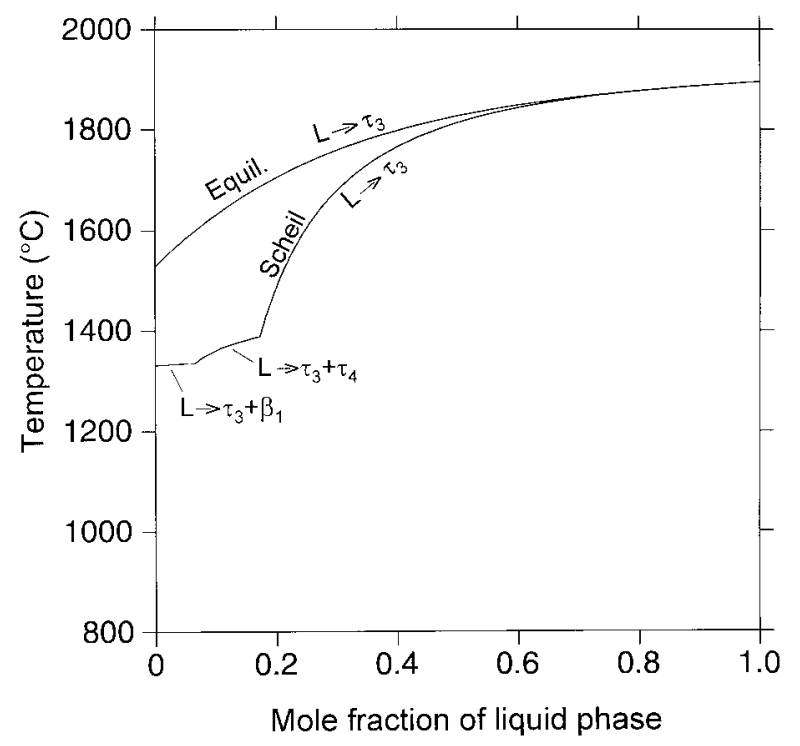

Fig. 14. Calculated molar fraction of the liquid phase versus temperature during solidification of the 39Al-29.5Ni-Ti (at \%) alloy under equilibrium and Scheil model conditions.

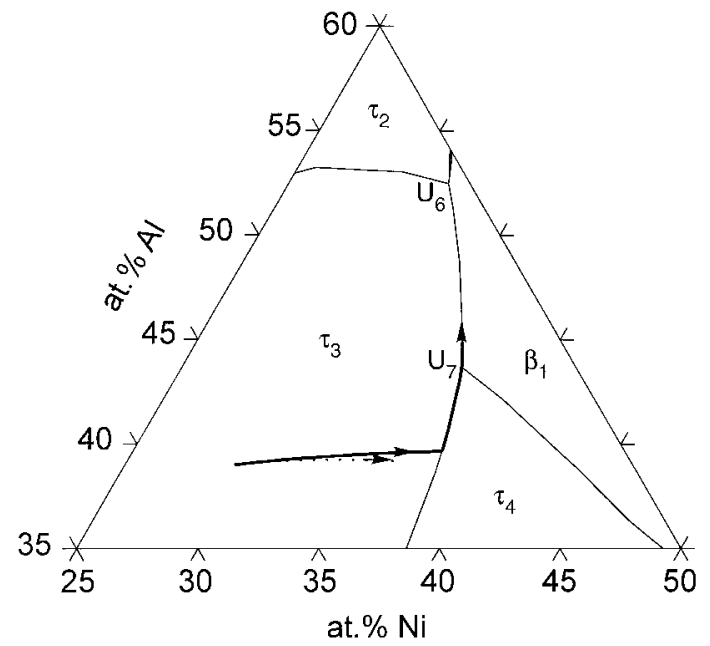

Fig. 15. Solidification path of the 39Al-29.5Ni-Ti (at \%) alloy under equilibrium (dotted line) and Scheil model conditions (thick lines), imposed on the calculated projection of the liquidus surfaces (thin lines).

prediction of the reaction $L \rightarrow \tau_{3}+\tau_{4}$ in Fig. 14. As to the predicted eutectic $L \rightarrow \tau_{3}+\beta_{1}$, no corresponding microstructure is visible in Fig. 2. This result indicates that the experimental solidification was probably under a condition between equilibrium and Scheil, which means that a partial back diffusion has occurred in the solid phases. This is understandable because the solidification proceeds at high temperatures $\left(>1330^{\circ} \mathrm{C}\right)$. Furthermore, the reaction $L \rightarrow \tau_{3}+\beta_{1}$ is predicted by the Scheil model to happen within a very narrow temperature range from 1335 to $1230^{\circ} \mathrm{C}$, compared to the temperature range of the entire solidification process $\left(1895-1230^{\circ} \mathrm{C}\right)$.

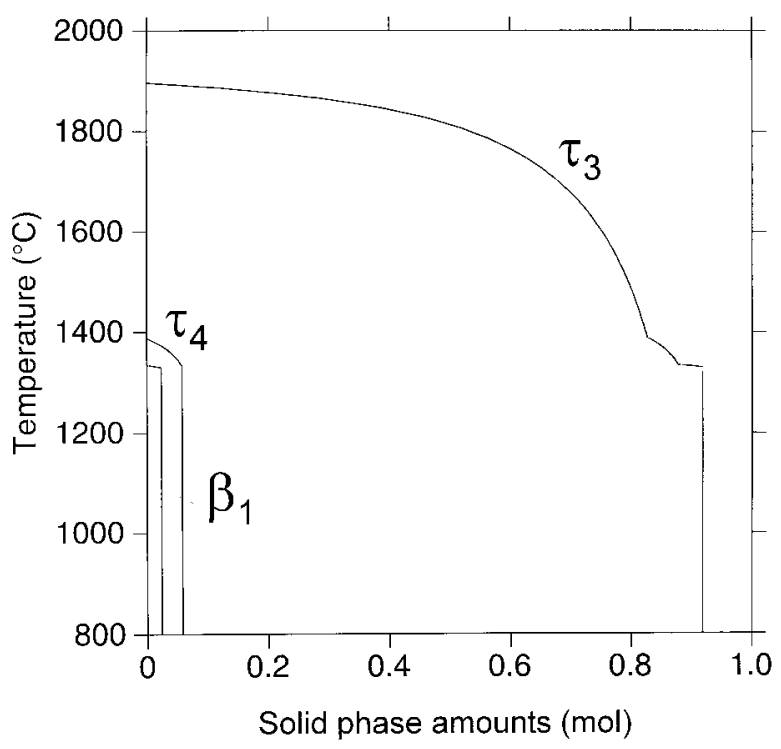

Fig. 16. Calculated molar fraction of the solid phases versus temperature during solidification of the 39Al-29.5Ni-Ti (at \%) alloy under Scheil model conditions.

The calculated compositions of the primary phase $\tau_{3}$ at the very beginning of solidification are $38.58 \mathrm{Al}-26.95$ $\mathrm{Ni}-34.48 \mathrm{Ti}(\mathrm{at} \%)$, almost the same as the experimental values 38.5Al-27Ni-34.5Ti (at\%) determined by EMPA in the core of the primary phase. At the end of the primary solidification, the compositions of the finally precipitated $\tau_{3}$ are predicted to be $39.37 \mathrm{Al}-30.23 \mathrm{Ni}-30.40 \mathrm{Ti}$ (at $\%$ ). Fig. 16 presents the phase fractions in the solidified alloy versus temperature under Scheil condition. It can be seen that in the solidified alloy there are 91.8 at $\% \tau_{3}$, 5.7 at $\% \tau_{4}$, and 2.5 at $\% \beta_{1}$. Such data may be helpful in controlling the microstructures of the cast alloys by choosing an appropriate composition and cooling rate. The higher the cooling rate, the greater the volume fraction of the secondary $\tau_{4}$ particles, or even some $\beta_{1}$ particles, in the $\tau_{3}$ matrix. On the other hand, the $\beta_{1}$ particles, or even $\tau_{4}$, can be avoided by a slower cooling rate.

\section{Conclusions}

A complete and internally consistent picture of polythermal ternary $\mathrm{Al}-\mathrm{Ni}-\mathrm{Ti}$ phase equilibria has been developed by thermodynamic modeling. It enables a quantitative check and comparison of different - though interrelated - data on melting and solid state phase equilibria in a wide temperature range of the entire ternary system. New experimental data are presented on phase equilibria at 800 and $1000^{\circ} \mathrm{C}$ and with the liquid phase. They have been combined with available literature data, including the extensive study at $900^{\circ} \mathrm{C}$ presented in the companion paper, part I, to form the basis of our modeling. An application to the simulation of the solidification behavior of a ternary alloy is also demonstrated. 
This may be useful in controlling the microstructure with reduced number of costly experiments, since this type of calculation can now be performed for any ternary alloy.

The limitations of the present modeling are partly due to conflicting experimental information and partly due to model simplifications. Especially the thermodynamic stability of $\tau_{4}$, which was fixed from a previous assessment of the Ni-rich equilibria [1], appears to be too low in comparison with the experimental Ni-poor equilibria. The model for $\tau_{3}$ is not reproducing the exceptional banana-shaped homogeneity region of $\tau_{3}$ at $900^{\circ} \mathrm{C}$. The metastability of $\tau_{3}$ at $800^{\circ} \mathrm{C}$ does not agree with experimental data. The calculated $\kappa+\tau_{2}$ equilibrium at $900^{\circ} \mathrm{C}$ is in conflict with the experimental $\mathrm{Al}_{2} \mathrm{Ti}+\tau_{3}$ equilibrium. These points show the area of specific future studies. The overall agreement with the vast majority of experimental data, however, indicates a reasonable reliability of the present assessment.

\section{Acknowledgements}

This work was supported by the German BMBF under grant number $03 \mathrm{~K} 72058$ and by the Austrian Ministry of Science under grant GZ 49.885/2-II/4/93 as part of the European Concerted Action COST 507, as well as by the Training and Mobility (TMR) Programme of the European Commission within the Clausthal Centre of Process Engineering Systems (COPES).

\section{References}

[1] Dupin, N. Contribution to the thermodynamic evaluation of multicomponent Ni-based alloys (in French), Ph.D. Thesis, Institut National Polytechnique de Grenoble, 1995, pp. 1-259.

[2] Huneau, B, Rogl, P. Zeng, K, Schmid-Fetzer, R, Bohn, M, Bauser, J. Intermetallics 1999;7(12):1337-45.

[3] Budberg, PB. In: Effenberg G, Petzow G, editors. Ternary alloys, Vol.8: Al-Ni-Tb to AL-Zn-Zr. Weinheim, Germany: VCH Verlagsgesellschaft, 1993. p. 7-21.

[4] Lee KJ, Nash PJ. Phase Equilibria 1991;12(5):551-62.
[5] Nash P, Liang WW. Metall Trans A 1985;16(3):319-22.

[6] Bauer J, Rogl P, Perrin A, Bohn M, Wolf W, Podloucky R, LeFriece Y, Antoine D. Intermetallics 1996;4(1):71-6.

[7] Dinsdale AT. CALPHAD 1991;15(4):317-425.

[8] Saunders, N. Thermodynamic evaluation of the Ni-Ti system. Internal Report, University of Surrey, 1993.

[9] Bätzner, C. Ph.D. Thesis, Universtät Stuttgart, Stuttgart, Germany, 1994.

[10] Kattner UR, Lin J-C, Chang YA. Metall Trans 1992;23A(8):2081-90.

[11] Saunders, N. Thermodynamic parameters characterising the phase diagrams of the $\mathrm{Al}-\mathrm{Ti}, \mathrm{Al}-\mathrm{V}$ and $\mathrm{Ti}-\mathrm{V}$ binary systems. Internal Report, University of Surry, 1990.

[12] Zhang F, Chen SL, Chang YA, Kattner U. Intermetallics 1997;5:471-82.

[13] Murray, J.L. In: Murray JL, editor. Phase diagrams of binary titanium alloys. Metals Park, OH, USA: ASM International, 1987. p. 12-24.

[14] McCullough C, Valencia JJ, Mateos H, Levi CG, Mehrabian R, Rhyne KA. Scr Metall 1988;22(7):1131-6.

[15] McCullough C, Valencia JJ, Levi CG, Mehrabian R. Acta Metall 1989;37(5):1321-36.

[16] Valencia JJ, McCullough C, Levi CG, Mehrabian RH. Scr Metall 1987;21(10):1341-6

[17] Kaltenbach K, Gama S, Pinatti DG, Schulze K. Z Metallkd 1989;80(7):511-14.

[18] Schuster JC, Ipser H. Z Metallkd 1990;81(6):389-96.

[19] Shull RD, Cline JP. High Temp Sci 1990;26:95-117.

[20] Mishurda JC, Perepezko H. In: Kim YW, Boyer RR, editors. Microstructure-property relationships in titanium-aluminides and alloys. TMS, Warrendale PA, USA. Proc Seven Sess Symp, 1990. p. $3-30$

[21] Anderson CD, Hofmeister WH, Bayuzick RJ. Metall Trans 1993;24A(1):61-6.

[22] Kainuma R, Palm M, Inden G. Intermetallics 1994;2(4):321-32.

[23] Ansara I, Dupin N, Lukas HL, Sundman B. J Alloys Compd 1997;247(1-2):20-30.

[24] Redlich O, Kister AT. Ind Eng Chem 1948;40:345-8.

[25] Hillert M, Staffanson LI. Acta Chim Scand 1970;24(10):3618-26.

[26] Mazdiyasni S, Miracle DB, Dimiduk DM, Mendiratta MG, Subramanian PR. Scr Metall 1989;23(3):327-31.

[27] Omarov AK, Seitzhanov SV, Idirisov AI. Izv Akad Nauk Kazakh SSR Ser Khim 1985;1:36-42.

[28] Markiv VY, Burnashova VV, Ryabov VR. Akad Nauk Ukr SSR Metallofiz 1973;46:103-10.

[29] Raman A, Schubert K. Z Metallkd 1965;56(2):99-104.

[30] Flemings MC. Solidification processing. New York: McGrawHill, 1974.

[31] Sinha AK. Progr Mater Sci 1972;15:79-185. 